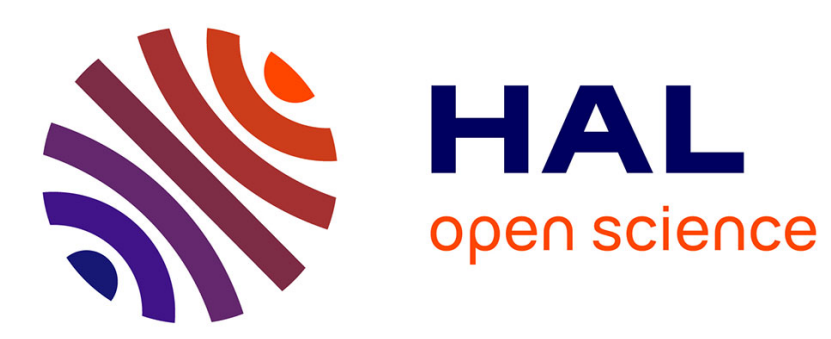

\title{
First-in-class matrix anti-assembly peptide prevents staphylococcal biofilm in vitro and in vivo
}

\author{
Rafael Gomes von Borowski, Sophie Chat, Rafael Schneider, Sylvie \\ Nonin-Lecomte, Serge Bouaziz, Emmanuel Giudice, Aline Rigon Zimmer, \\ Simone Cristina Baggio Gnoatto, Alexandre José Macedo, Reynald Gillet
}

\section{To cite this version:}

Rafael Gomes von Borowski, Sophie Chat, Rafael Schneider, Sylvie Nonin-Lecomte, Serge Bouaziz, et al. First-in-class matrix anti-assembly peptide prevents staphylococcal biofilm in vitro and in vivo. 2021. hal-03383344

\section{HAL Id: hal-03383344 https://hal.science/hal-03383344}

Preprint submitted on 18 Oct 2021

HAL is a multi-disciplinary open access archive for the deposit and dissemination of scientific research documents, whether they are published or not. The documents may come from teaching and research institutions in France or abroad, or from public or private research centers.
L'archive ouverte pluridisciplinaire HAL, est destinée au dépôt et à la diffusion de documents scientifiques de niveau recherche, publiés ou non, émanant des établissements d'enseignement et de recherche français ou étrangers, des laboratoires publics ou privés. 
First-in-class matrix anti-assembly peptide prevents staphylococcal biofilm in vitro and in

${ }^{1}$ Univ. Rennes, CNRS, Institut de Génétique et de Développement de Rennes (IGDR), UMR6290,

9 Universidade Federal do Rio Grande do Sul, 90610-000 Porto Alegre, Brazil; ${ }^{3}$ Université de Paris,

11 Pharmacie, 75006 Paris, France; ${ }^{4}$ Centro de Biotecnologia da Universidade Federal do Rio Grande do

14 *To whom correspondence may be addressed. Email reynald.gillet@univ-rennes1.fr or

15 alexandre.jose.macedo@gmail.com.

\section{Abstract}

Staphylococci are pathogenic biofilm-forming bacteria, source of multidrug-resistance and/or tolerance causing a broad spectrum of infections. These bacteria are enclosed in a matrix that allows them to colonize medical devices such as catheters and tissue, and which protects against antibiotics and immune systems. Advances in antibiofilm strategies for targeting this matrix are 

antibiotic, carbohydrate-binding peptide.

Keywords: antibiofilm, biofilm, matrix, peptides, anti-assembly, resistance, tolerance.

Introduction

Antimicrobial failure is a worldwide challenge, currently addressed by a WHO global action plan (1). A lack of new antibiotics and the inappropriate use of older treatments mean that multidrug-resistant strains are increasing (2). This process is favored by biofilm development, and microorganisms enclosed in biofilm matrix have antibiotic resistance that is up to 1000 times higher than planktonic ones (3). This makes the matrix itself an important target for biofilm control. Biofilms are organized microbial clusters made of a self-assembled matrix that usually attaches to a surface, whether abiotic (medical devices such as catheters, teeth, etc.) or biotic (host tissues, mucus, chronic wounds, etc.) increased tolerance and resistance to antibiotics, disinfectants, and host defenses $(6,7)$. Other advantages over planktonic forms include physiological and biochemical changes, beneficial quorum sensing, higher (up to 100 times) mutation rates, and persister cell development (8-10).

Staphylococci are the most frequent sources of nosocomial infections, particularly S. epidermidis and S. aureus. While S. aureus expresses many virulence factors such as toxins and proteases, for $S$. 
infection-causing disease (12), surviving on various surfaces for months (13). It is present in $30 \%$ of health care-involving bloodstream infections, and is significantly associated with medical device infections, including $15-40 \%$ of prosthetic valve endocarditis (14) and $30-43 \%$ of prosthetic orthopedic device infections (15). More than 150 million intravascular catheters are used per year in the USA, and there are about 250,000 catheter-related infections $(16,17)$. These bacteria are developing antibiotic multi-resistances such as elevated glycopeptide minimal inhibitory concentrations (18), and $73-88 \%$ of isolates display resistance to oxacillin, fluoroquinolones, macrolides, clindamycin, and trimethoprim/sulfamethoxazole (19-21).

In this circumstance, the extracellular matrix is a complex physicochemical barrier representing one of the biggest challenges in microbial treatments (4). Therefore, the development of antivirulence strategies such as antibiofilm agents is crucial for the current antibiotic crisis, and peptides are an increasingly arsenal for controlling pathogenic biofilms (22-24).

Here, we describe the discovery of the antibiofilm peptide capsicumicine, inspired by natural peptides from the seeds of the red pepper Capsicum baccatum. We elucidated its mechanism of action (MoA), the matrix anti-assembly (MAA). Capsicumicine is the first-in-class non-antibiotic peptide displaying this extracellular MoA. Notably, we report an in vivo anti-infective proof of concept towards to the use of capsicumicine for complementary treatment of infectious diseases.

Capsicumicine prevents biofilm formation without antibiotic activity. We synthesized three peptides inspired by a natural antibiofilm fraction previously identified from Capsicum baccatum var. pendulum pepper seeds (25): P1 (RVQSEEGEDQISQRE), P2 (RAEAFQTAQALPGLCRI), and P3 (RSCQQQIQQAQQLSSCQQYLKQ). To find the most active one, we exposed these compounds to strong biofilm-forming S. epidermidis RP62A (ATCC 35984). After $24 \mathrm{~h}$, crystal violet was used to 
quantify the remaining biofilm (Fig. 1A). The P3, named "capsicumicine," was the most active with

particularly strong antibiofilm activity. Biofilm decreases were observed at all tested concentrations,

but especially at $10 \mu \mathrm{M}$. There, biofilm was reduced by over $91 \%$, independently of cell growth

inhibition (Fig. 1B). To examine the effects of capsicumicine on growth, we checked S. epidermidis

colony-forming unit (CFU) counts after peptide exposure. As expected, the CFUs were unchanged by capsicumicine, so the peptide's biofilm inhibition is not due to bactericidal activity (Fig. 1B). To verify

the interactions between capsicumicine and established matrices, we exposed a pre-existing $S$.

epidermidis biofilm to a single concentration $(100 \mu \mathrm{M})$ of the peptide. At that concentration,

capsicumicine accounts for $15 \%$ of the disruption of pre-existing biofilm (Fig. S1).

A

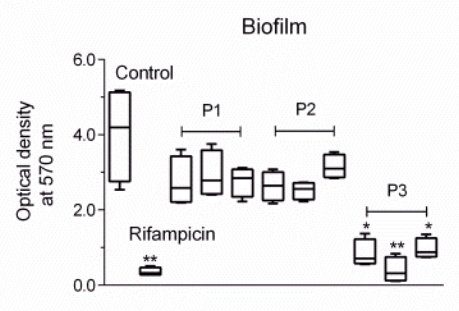

C

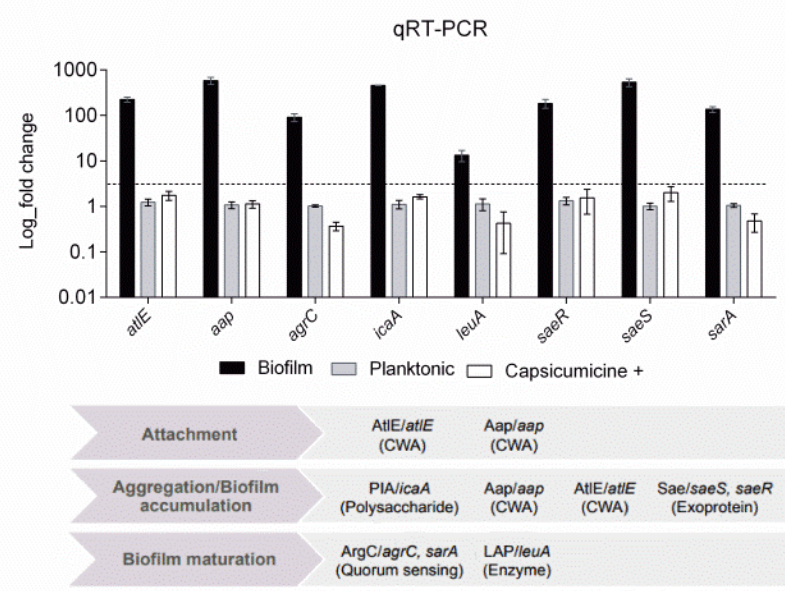

B

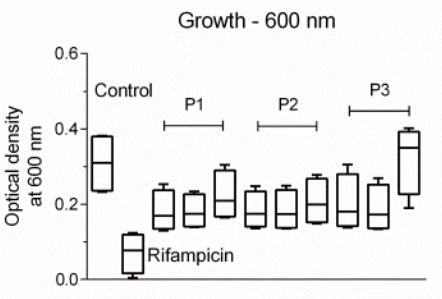

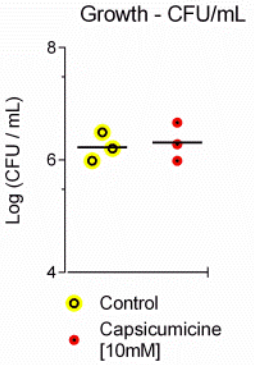

D

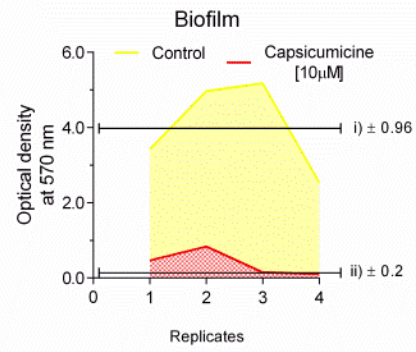

Cytotoxicity of Capsicumicine

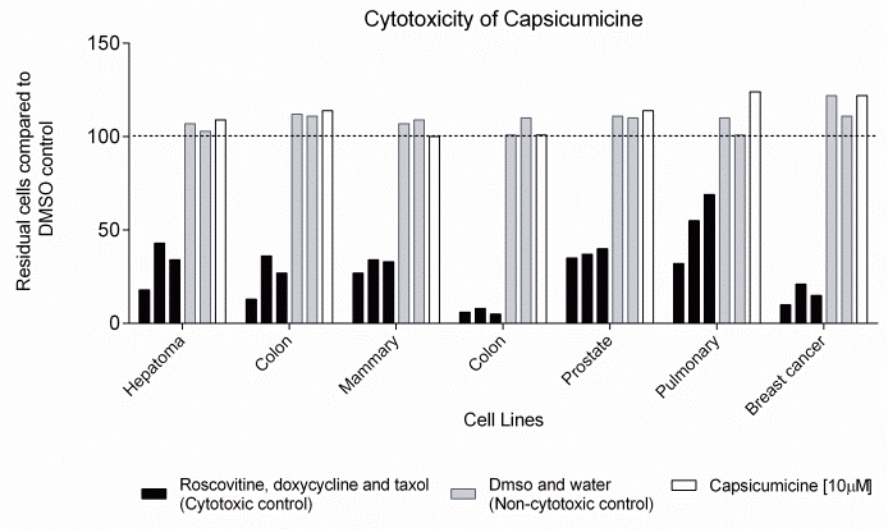

Fig. 1. Antibiofilm activity of bioinspired peptides and cytotoxicity. (A) Antibiofilm activity of peptides P1, P2, and P3 (capsicumicine) at 1, 10, and $100 \mu \mathrm{M}$. Quantification of Staphylococcus epidermidis (ATCC 35984) biofilm and growth were done at an optical density; bacteria without peptide exposure (Control) and the antibiotic control, $16 \mu \mathrm{g} / \mathrm{mL}$ rifampicin (Rif.). Student's $t$-test: ${ }^{*}, p \leq 0.05 ;{ }^{* *}, p \leq$ 0.01 . (B) Antibiofilm activity and growth of capsicumicine at $10 \mu \mathrm{M}$. Yellow represents $100 \%$ of formed biofilm and red represents the remaining biofilm after exposure to capsicumicine. Growth 

associated with extracellular matrix components, not entering into bacterial cells or the walls or

was verified using colony-forming units (CFUs). (C) Gene expression (mean log fold changes \pm standard errors of the means) of the encoding genes involved in S. epidermidis biofilm formation as compared to the planktonic (grey) and biofilm controls (black), with the ssrA gene used as a reference. The group exposed to capsicumicine is shown in white. CWA, cell wall-anchored proteins. $D$ ) Capsicumicine cytotoxicity evaluation in representative human cell lines shown via automated image-based cellular content analysis. Cell counts are presented as residual cell percentages (\%) compared to the average of the DMSO control, with water control also shown (grey bars). The black bars on the left show cytotoxic controls (roscovitine, doxycycline, and taxol), while the white bars are cells exposed to $10 \mu \mathrm{M}$ of capsicumicine.

To study the peptide's possible mechanisms of action, we selected several genes involved in different stages of biofilm development (at/E, aap, agrC, icaA, leuA, saeR, saeS, and sarA); primers are listed in Table S1. Fold changes were analyzed by quantitative real-time PCR (qRT-PCR). Since exposed bacteria remain planktonic, we compared their relative gene expressions to planktonic control cells. For all tested genes, capsicumicine-exposed cells show the same fold changes as the control (Fig. 1C).

Capsicumicine is not cytotoxic in mammalian cells. To ensure that capsicumicine is safe before propose in vivo trials, we verified its cytotoxicity in seven different representative human cell lines. We used automated image-based cellular content analysis and found that capsicumicine-treated cells perform exactly the same as untreated controls, displaying no cytotoxicity (Fig. 1D).

\section{Independently of cell interactions, capsicumicine impairs biofilm attachment, aggregation, and}

accumulation. To explore its activity during the first stages of biofilm development, we analyzed biofilm cultures on polystyrene coupons with and without capsicumicine after 1, 4, and $24 \mathrm{~h}$.

Scanning electron microscopy (SEM) analysis shows that bacterial attachment decreases after $1 \mathrm{~h}$ of capsicumicine exposure, with biofilm accumulation and cell aggregation profiles strongly reduced after 4 and $24 \mathrm{~h}$ (Fig. 2A). This demonstrates that capsicumicine prevents S. epidermidis coupon adhesion, and notably this activity still occurs after $24 \mathrm{~h}$ incubation. In order to localize the peptide, we used capsicumicine conjugated to fluorescein isothiocyanate (capsicumicine-FITC) and confocal fluorescence microscopy (CFM). Analysis of the CFM images showed that capsicumicine-FITC stays membranes (Fig. 2B). 
A

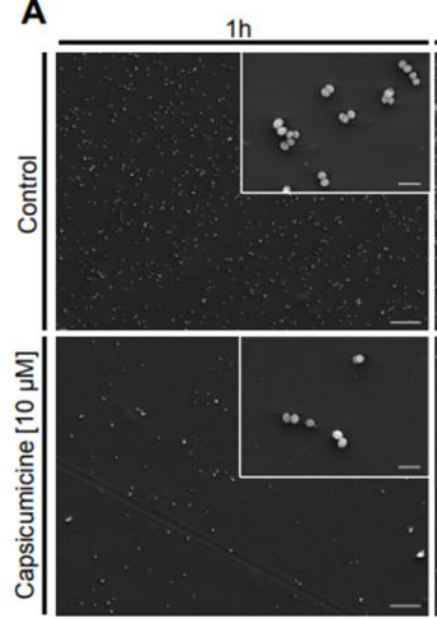

B
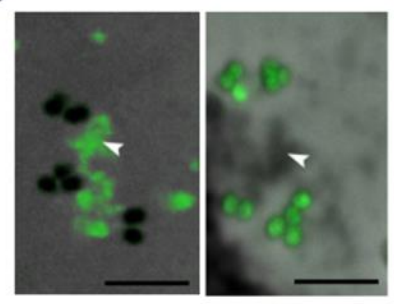
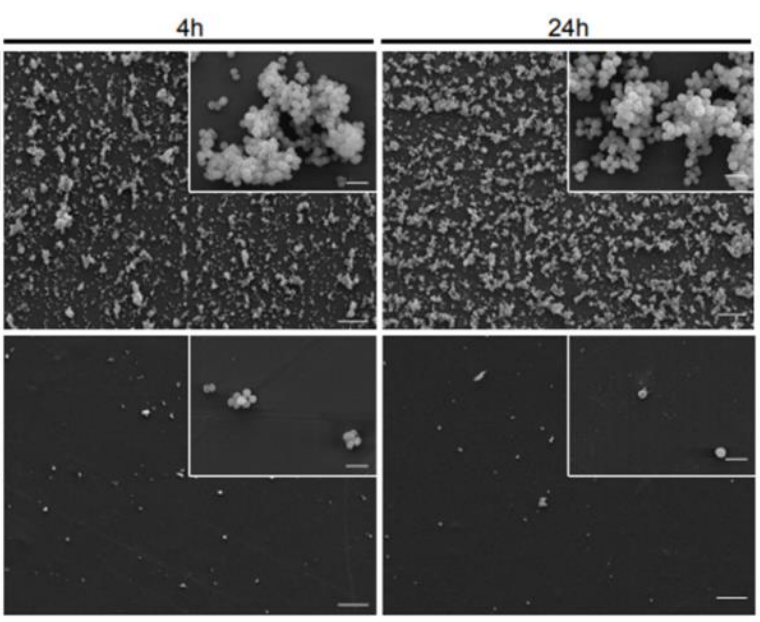

C
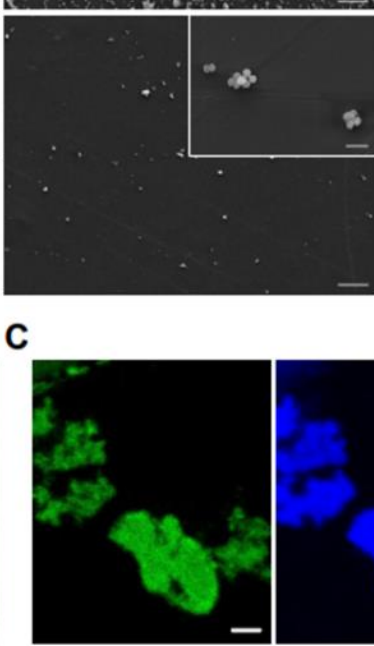
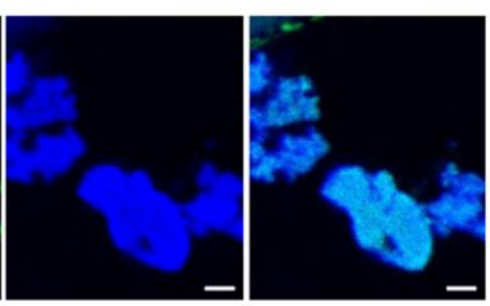
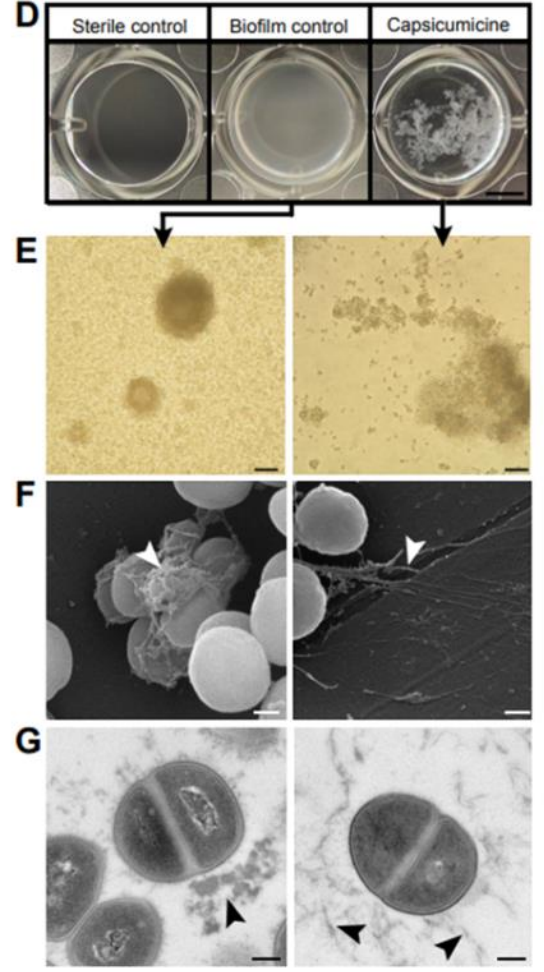

Fig. 2. Biological characterization of capsicumicine by microscopic approaches. (A) SEM images of polystyrene coupons after 1, 4, or 24h of culture with Staphylococcus epidermidis (ATCC 35984). Top: peptide-less biofilm control; bottom: cultures exposed to $10 \mu \mathrm{M}$ capsicumicine. Magnification $\times 500$, with insets at x5,000; scale bars, $10 \mu \mathrm{m}$. (B) CFM images: (left) S. epidermidis after exposure to $10 \mu \mathrm{M}$ capsicumicine-FITC, with the matrix in green (fluorescence), and the bacterial cells in black (no fluorescence); (right) control, S. epidermidis after exposition to pseudonajide-FITC, an antibacterial peptide, this time showing the matrix in gray (no fluorescence) and bacterial cells in green (fluorescence). White arrows indicate matrix content. Scale bars, $5 \mu \mathrm{m}$. (C) CFM images: (left) $S$. epidermidis after exposure to $10 \mu \mathrm{M}$ capsicumicine-FITC; (center) after exposure to concanavalin $A$ conjugates; and (right) their colocalization. Scale bars, $5 \mu \mathrm{m}$. (D-G) These images explore the organizational state of S. epidermidis biofilm after $24 \mathrm{~h}$ in the presence (right) or absence (control, left) of capsicumicine. (D) Macroscopic examination by pictures from the bottom of 24-well plates. The "sterile control" shows no bacteria or biofilm formation; the "biofilm control" shows homogenous adhered layers of bacteria; and "capsicumicine" shows non-adhered bacteria but agglutinates; scale bar, $5 \mathrm{~mm}$. (E) TLM images show the biofilm control with overlapping attached bacterial clusters, while the capsicumicine-exposed culture shows agglutinated non-adhered cells; scale bar, $20 \mu \mathrm{m}$. (F) SEM images show the control with dense globular-like matrix features, while the capsicumicine-exposed culture shows fibrillary branch-like oligomer structures; scale bar, $200 \mathrm{~nm}$. $(G)$ TEM images of the biofilm control show denser assembled structures, while the capsicumicineexposed displays thin fibrillary oligomer structures; scale bar, $200 \mathrm{~nm}$. Arrows indicate the matrices.

140 Capsicumicine disturbs S. epidermidis matrix assembly. Since capsicumicine's antibiofilm activity

141 was not associated with direct bacterial interactions or gene expression modulation, we used various

142 microscopic approaches to investigate the interactions between the peptide and the extracellular 
143 matrix. In the biofilm control, macroscopic observation shows a homogenous whitish adhered layer

144 covering the walls and bottoms of the well (Fig. 2D, middle), but when capsicumicine is present, we

145 see whitish flocculent non-adhered heterogeneous agglutinates (Fig. 2D, right). Transmitted light

146 microscopy (TLM) images match with these macroscopic observations (Fig. 2E). Crucially, SEM and

147 TEM technique yield ultra-structural descriptions that support these results, with the control biofilm

148 matrix showing denser assembled globular-like structures, while the capsicumicine-exposed matrix is

149 less dense and has thin fibrillary branch-like structures (Fig. $2 F$ and $G$ ). However, cellular

150 morphologies are not different from the controls. Therefore, different imaging techniques prove that

151 matrix assembly changes when capsicumicine is present.

152 Capsicumicine interacts with matrix polysaccharides. To explore the peptide's affinity with these

153 major matrix components, we exposed S. epidermidis cultures to capsicumicine (-FITC; green) and

154 saccharides staining, then analyzed them all by CFM. We used concanavalin A and calcofluor to

155 selectively target matrix saccharides (blue). Amounts of capsicumicine-FITC appear exclusively on the

156 matrix (Fig. $2 B$ left; S2B) and its colocalization indicates the interaction between both marked

157 elements (Fig. 2C, right; S2A). Visualization was done by individual (Fig. 2C, left and center) and

158 colocalization fluorescence (Fig. 2C, right). The peptide control was pseudonajide-FITC, an

159 antibacterial peptide; it showed green fluorescence in the cells but not in the matrix (Fig. 2B right;

160 S2C). To explore whether capsicumicine features are compatible with carbohydrate-binding modules

161 (CBMs), we performed a BLAST and amino acid alignments between capsicumicine and chitin-,

162 chitosan-, and polysaccharide intercellular adhesin (PIA), PIA-binding proteins (Table S2). UniProt

163 tools (26) and CAZy information crossing (27) showed that capsicumicine does in fact present CBM

164 homology with all tested proteins (Fig. S3).

165 Capsicumicine shifts Staphylococcal synthetic matrix. To confirm these interactions in the absence

166 of bacteria metabolic or regulatory influences, we adapted a model of artificial staphylococcal biofilm

167 assembly to test it. Briefly, we monitored the real-time molecular self-assembly (RTMSA) reaction by 
A
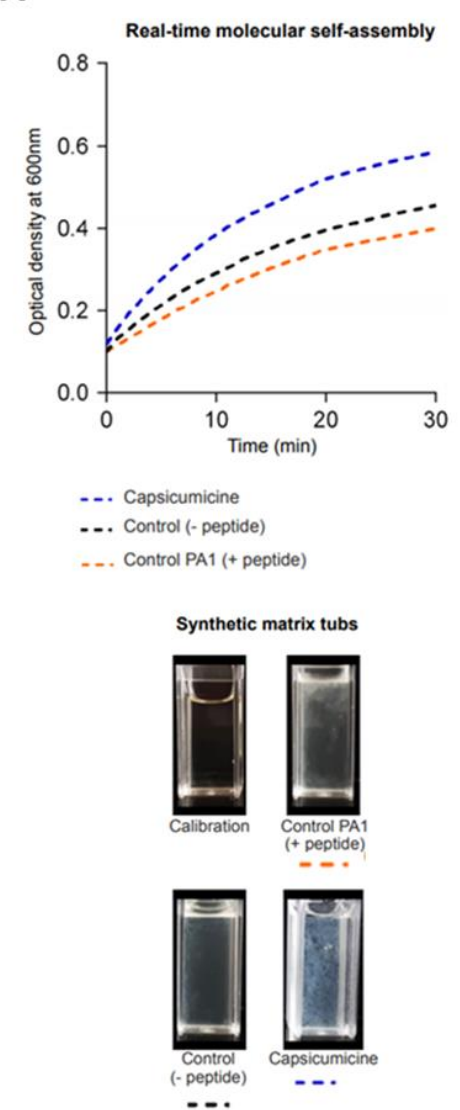

B

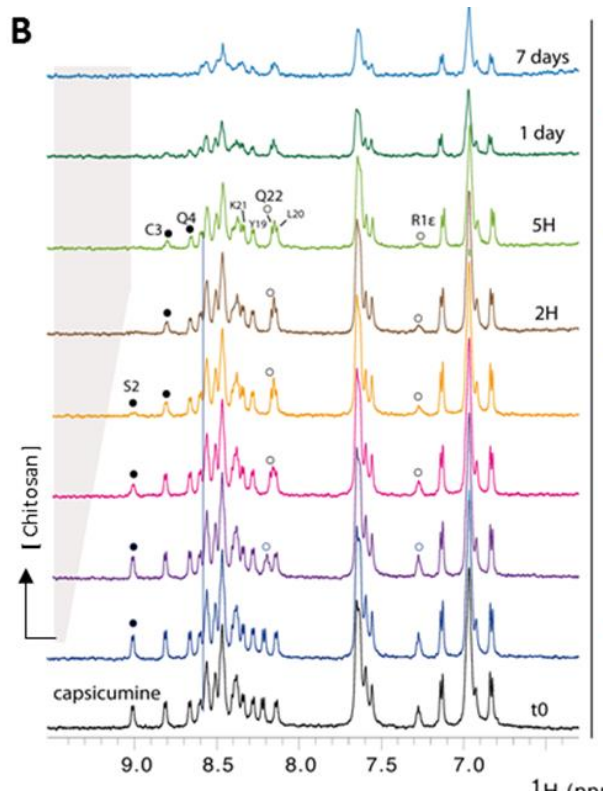

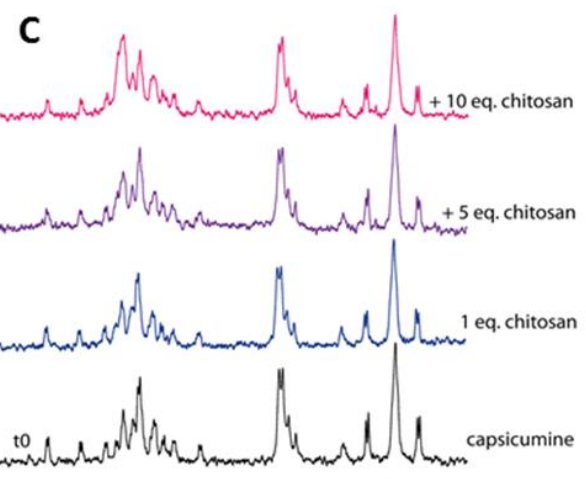
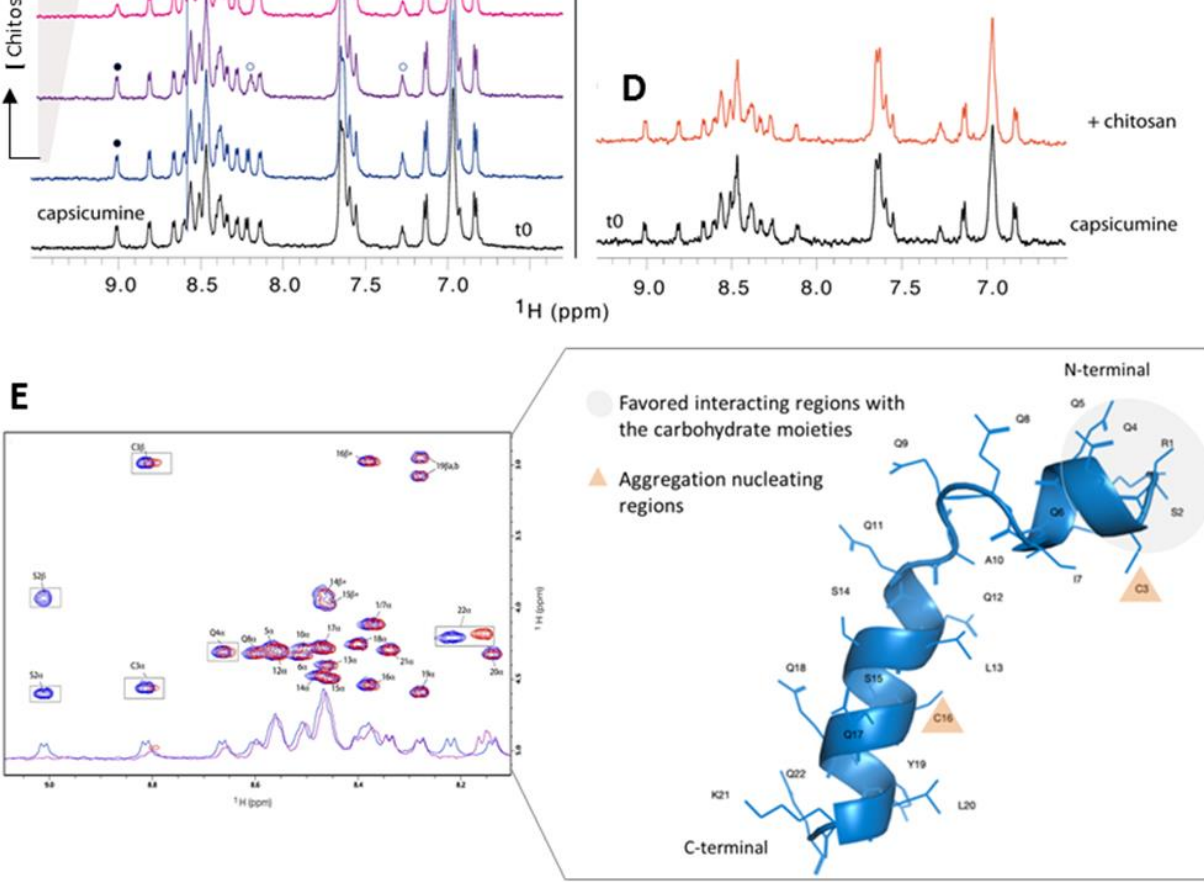

Fig. 3. Molecular interactions between capsicumicine and target-saccharides. (A) RTMSA curves of artificial staphylococcal biofilm assembly. Optical densities $\left(\mathrm{OD}_{600}\right)$ were recorded as a function of the time in the presence of capsicumicine (blue dots) or PA1 as peptide control (orange dots) and the synthetic matrix without peptides (black dots); each reaction tub is shown under the graph. (B-D) NMR titration of capsicumicine by chitosan at $600 \mathrm{MHz}, 10^{\circ} \mathrm{C}$. (B) From the bottom to the top: increasing concentrations of soluble chitosan [ $<0.5$ eq. and $8 \%$ of volume variation] were gradually added to capsicumicine water solution. Specific line broadenings and frequency shifts are shown in the NMR spectrum for some amide protons upon titration; white bullets for terminal R1 and Q22 and 
black bullets for S2, C3 and Q4. (C) Assembled chitosan gradually added to soluble capsicumicine [0.5 eq.]; an overall line broadening was observed. (D) Assembled chitosan suddenly added to soluble capsicumicine [0.5 eq.]; the orange spectrum was recorded after few minutes of stirring. (E) Superposition of capsicumicine 1D NMR and TOCSY spectra immediately after sample preparation (blue) and chitosan [10 eq.] addition (red) and; capsicumicine structure predicted by Phyre2 server; according to NMR results the aggregation nucleating regions are shown as orange triangles and favored interacting region with sugar moieties is shown in grey.

To evidence the interactions between capsicumicine and target-saccharides we performed NMR

titration experiments using chitosan as a mimic of the matrix poly-N-acetyl glucosamine (PNAG). The evolutions of the capsicumicine NMR spectra upon chitosan addition were monitored. Reference 1D

194 proton NMR spectra were recorded at all tested conditions. Chitosan was gradually added from a

195 concentrated solution and spectra changes were monitored. This solution remained clear during the

1967 days of recording. The addition of soluble chitosan drives noticeable changes of the resonance

197 frequency of some of the amide protons mainly in the N-terminal part of the peptide $<$ S2, C3, Q4 and R1 (not shown) > (Fig. 3B). Conversely, to < Y19, L20 and K21 NH and the Y19 > aromatic proton resonances, between 6.8 and 7.2 are not sensitive to the presence of chitosan (Fig. 3E). The concentration variation of chitosan was estimated to less than $0.5 \mathrm{eq}$ at the end of the titration. This small quantity of chitosan drives spectral modifications in the $\mathrm{N}$-terminal part of capsicumicine and strongly supports the interaction between both partners. The important broadening observed after 1 day shows that the peptide structure keeps evolving over time and this is not due to cysteines' 204 oxidation (Fig $4 D, E$ ). These experiments were repeated adding gelled chitosan instead of soluble 205 chitosan, mimicking an assembled matrix. It does not drive any significant spectral changes, except 206 for a general line broadening which probably arises from an increased viscosity of the solution (Fig. $2073 C$ ). The suddenly addition of gelled chitosan pellets in the NMR solution, does not modify the 208 spectrum either (Fig 3D).

210 understand the mechanisms involved in capsicumicine bioactivity we step toward the knowledge of 211 its conformation in solution and molecular interactions with matrix representative saccharides. To 
212 monitor the conformation of free capsicumicine in response to time and temperature we first used

213 Circular Dichroism (CD) and Nuclear Magnetic Resonance (NMR) spectroscopies. Spectral

214 deconvolution using the CDSSTR algorithm discloses a helical structure (about 43-44\%) with a non-

215 negligible proportion of $\beta$ strands (31-33\%) and unfolded (about 20\%) (Table S3). The structure

216 stability was assessed over 5 days recording the $C D$ spectra at $5^{\circ} \mathrm{C}$ (Fig. $4 \mathrm{~A}$ ). The main structure

217 observed over the first $24 \mathrm{~h}$ is helical. After 5 days, deconvolutions show a slight decrease of the

218 helical content. Furthermore, on the fifth day, CD spectra were also recorded at 15 and $30^{\circ} \mathrm{C}$ (Fig.

$2194 B$ ). The helical content decreases to $18-23 \%$ while the $\beta$ strand and turn mean proportions rose

220 around $37 \%$ and $23 \%$, respectively. The proportion of unfolded structures also increased. After 2

221 days at room temperature, a new $C D$ spectrum was recorded at $30^{\circ} \mathrm{C}$. The helix proportion became

222 less than $15 \%$ in favor of $\beta$ strand and unfolded conformations (respectively $38-41 \%$ and $31-35 \%$ ).

223 During these days, the peptide solution remained clear, showing no macroscopic signs of

224 aggregation.

225 The 1D and 2D NMR spectra of a $0.3 \mathrm{mM}$ freshly prepared solution of capsicumicine were recorded at

$22610^{\circ} \mathrm{C}, \mathrm{pH}$ 5.0. All amide resonances were unambiguously assigned using TOCSY and NOESY spectra,

227 except for Q9 and Q11 residues (Fig. S4). The spectral dispersion and the spreading of the amide

228 proton resonances disclose that the peptide is folded with one conformation in these conditions. In

229 accordance with CD spectra, a second set of $\mathrm{NH}$ resonances appears as a function of time (Fig. 4C, D),

230 revealing conformational changes in the slow exchange regime on the NMR time scale. After 5 days,

231 the most important chemical shift perturbations observed on the TOCSY spectrum are clustered

232 around the two cysteines apart from the two terminal residues (boxed cross-peaks, Fig. 4E).

233 Interestingly, 17 located one helix turn apart C3 is among the most sensitive residues to the

234 conformational perturbation. This is due to a disulfide bridge (DB), since no reducing agent was

235 added. Consequently, large proton chemical shift perturbations (CSP) would be expected for every -

$236 \mathrm{NH}$ amino acid and this was not detected (Fig. 4D, E). The prevalence of the second conformation 

recording time.

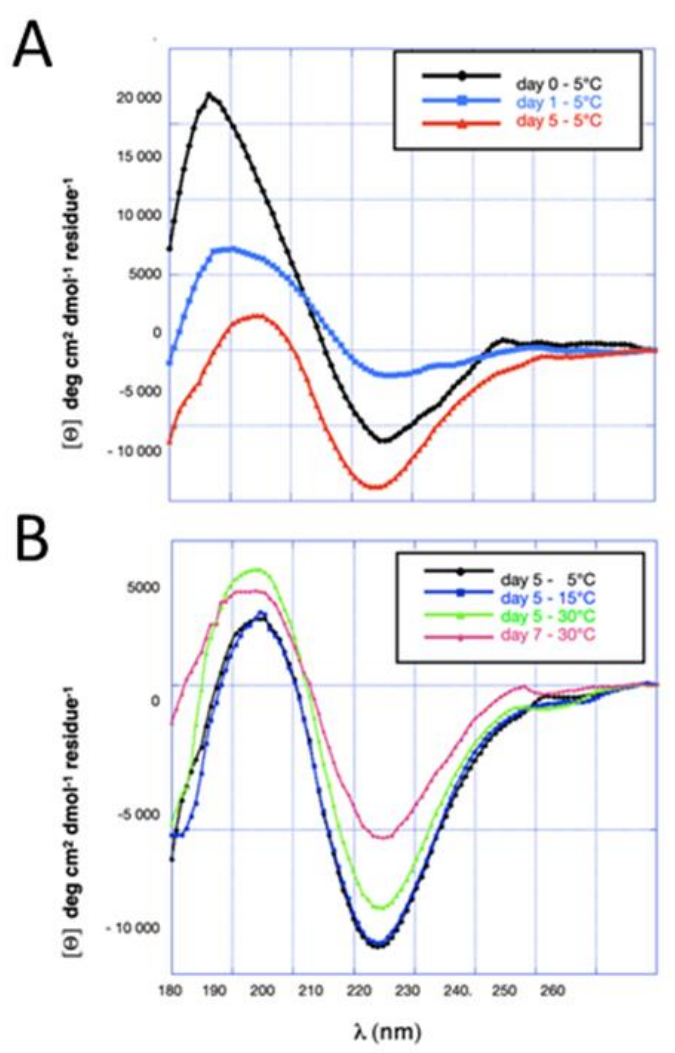

\section{D}

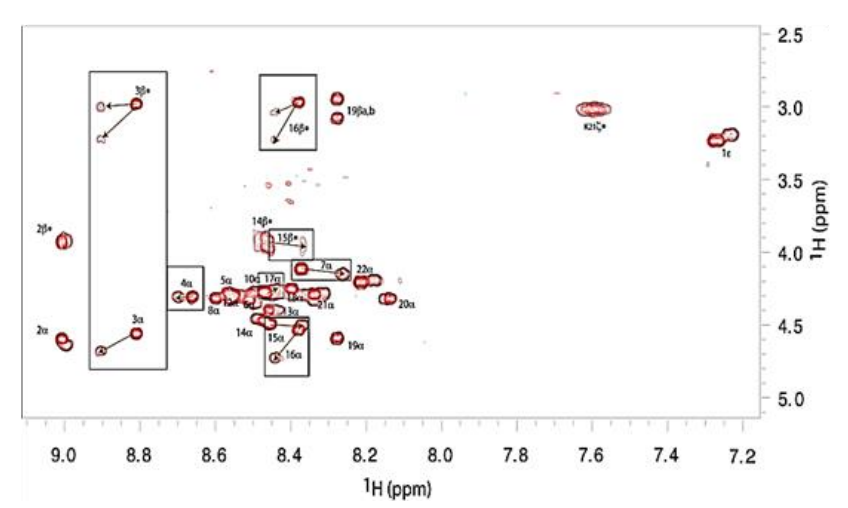

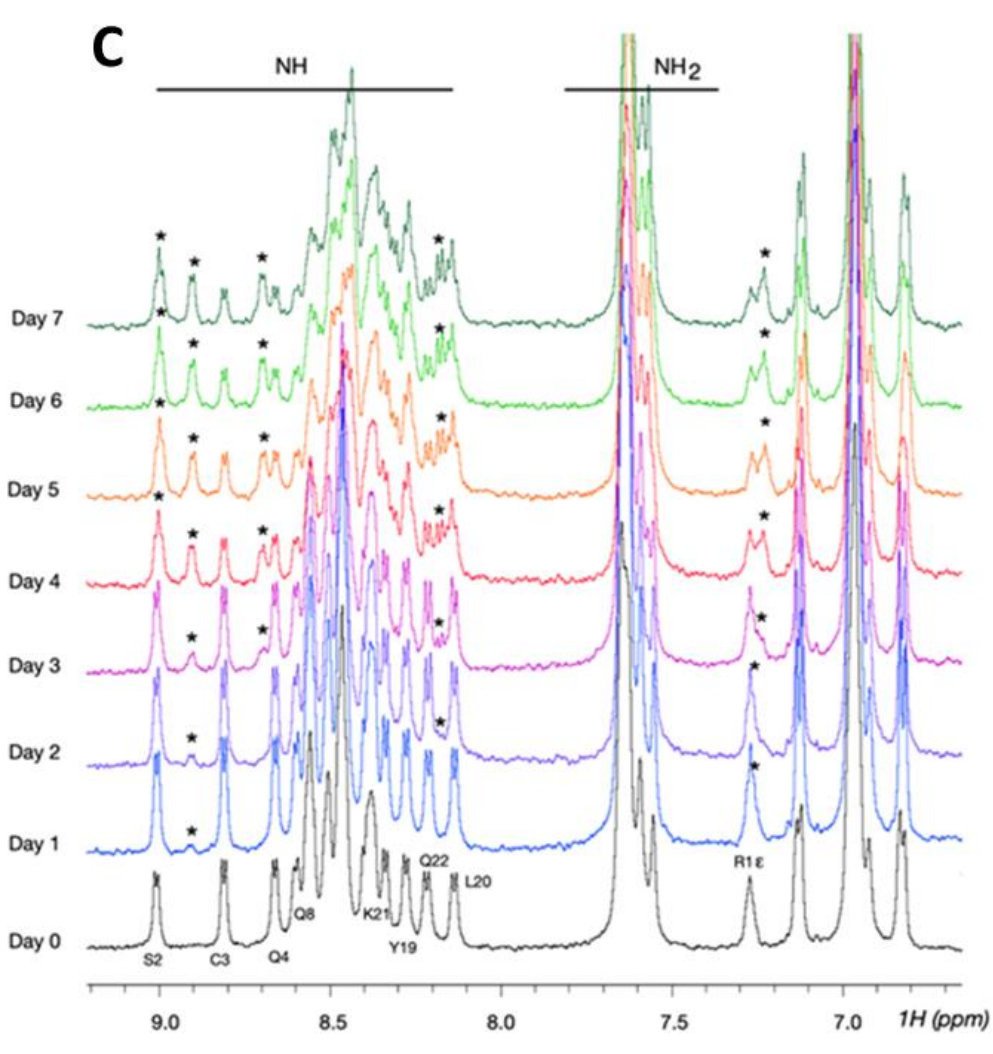

$\mathbf{E}$

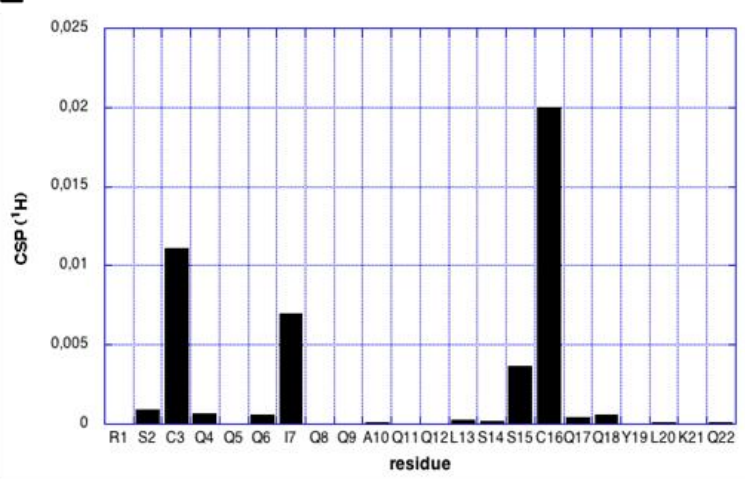

Fig. 4 Capsicumicine conformational changes. $C D$ spectras (A) Evolution at $5^{\circ} \mathrm{C}$ over 5 days; $(B)$ Evolution vs time and temperature. (C) NMR spectra recorded at $\mathrm{pH} 5.0,0.3 \mathrm{mM}$ and $10^{\circ} \mathrm{C}$ immediately after extemporaneous preparation (bottom); a second conformation appears over the time corresponding to the second set of amide resonances (stars). (D) Superposition of capsicumicine TOCSY spectra immediately after sample preparation (red) and at day 5 (brown). The most important chemical shit perturbations between the original and the new conformations are boxed and clustered around the two cysteines. (E) Proton chemical shift perturbations (CSP) computed from the TOSCY spectra are displayed in the box. 
Capsicumicine attenuates the dynamics of $S$. aureus (Xen36) infection on central venous catheter

250 (CVC). We performed a translational proof-of-concept evaluating long-term bacterial biofilm and 251 related infection in mice implanted with capsicumicine pre-coated CVC. These CVCs were previously 252 coated using an immobilization polymer ("hydrogel”) encompassing capsicumicine. This coating 253 confers amorphous and biocompatible surfaces to CVC (Fig. 5A). They were first validated in vitro, 254 decreasing $\geq 51 \%$ of $S$. aureus colonization (Fig. $5 B$ ). Then, bacterial development was evaluated in 255 vivo by bioluminescence imaging and bacterial load of harvested CVC (Fig. 5C-F). Two days (D2) after 256 S. aureus systemic infection, $40 \%$ of the control group (CVC-Hydrogel) presented high 257 bioluminescence signal (red zones) related to ROI against none in the treated group (CVC-Hydrogel + 258 capsicumicine), (Fig. 5C). Four days (D4) after infection, $75 \%$ of the control presented high ROI red 259 zones against $20 \%$ in the treated group (Fig. 5C). In addition, at D4, 1 animal was found dead in the 260 control group. Therefore, bioluminescence quantifications show that the treated group decreased $26156 \%$ (D2) and 54\% (D4) of the total flux compared to the control (Fig. 5D). This trend was also 262 observed on the CFU load at D4 (Fig. 5E). After image acquisitions (D4), 2 animals / group were 263 euthanized due to ethical criteria. At the end of the experiment, 7 days after infection (D7), the 264 treated group showed a decrease of $86 \%$ of bacterial load compared to control (Fig. 5E). Macroscopic 265 observation revealed that one animal from the control presented several organs with necrosis (liver, 266 spleen, intestine, kidneys and bladder). Finally, the treated group increased survival rate of $50 \%$ at 


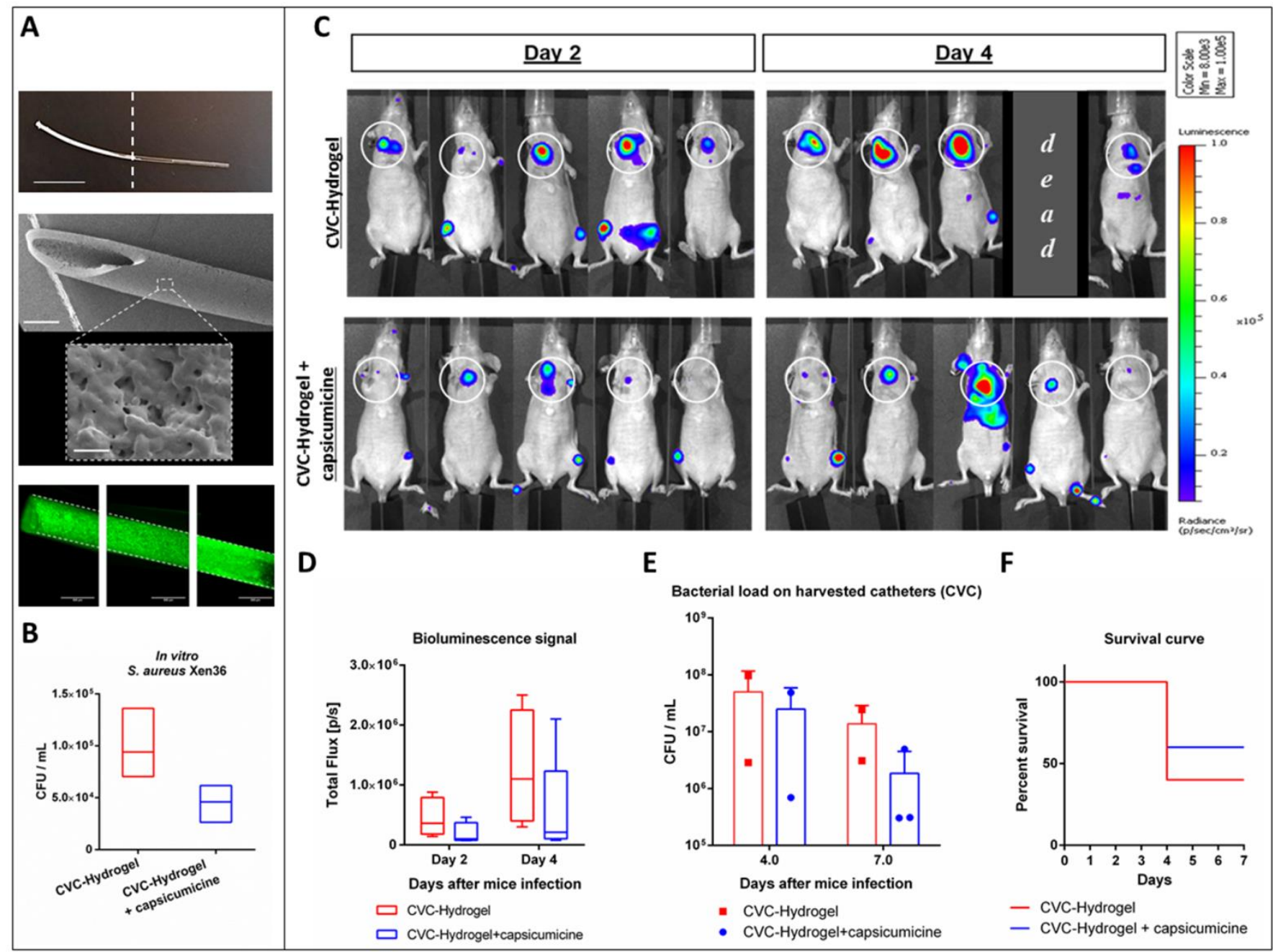

Fig. 5. Assessment of capsicumicine pre-coated central venous catheter (CVC) in mice (SKH1) infected by Staphylococcus aureus Xen36. (A-B) In vitro CVC development; (A) From the top to the bottom: a picture of a up to half capsicumicine pre-coated CVC (whitish), scale bar $1 \mathrm{~cm}$; SEM image of precoated CVC, scale bar $0.5 \mathrm{~mm}$ and its magnification, scale bar $1 \mu \mathrm{m}$ and; a compilation of images of fluorescence microscopy of pre-coated CVC (capsicumicine-FITC in green); (B) Bacterial load on CVC. (C-F) In vivo CVC assessment; (C) IVIS images (radiance-thresholded and smoothed with a bidimensional Gaussian filter) of ventral position for each mouse at each time point; the control group (CVC-Hydrogel) is shown in the top and the treated (CVC-Hydrogel + capsicumicine) in the bottom; white circles delimitate the CVC localization; the luminescence scale is presented in radiance. $(D)$ Bioluminescence quantification (ROI mean signals $\pm S D$ ) is shown as photons total flux $[\mathrm{p} / \mathrm{s}]$ for each group at each time point. (E) Bacterial load (CFU/mL) on harvested CVC for each mouse at each time point (day 4 and 7 after infection) and; $(F)$ The Kaplan-Meier survival curve (\% of survival) at each group, during 7 days after infection. All bioluminescence data and analysis were performed using IVIS Spectrum of Perkin Elmer. All experiments were conducted in accordance with ethical committee and the French authorities ( $n=5 /$ group). Bioluminescence images are presented with a defined pseudo-color scale to visualize the intensity of signals emitted. 
Bioinspired peptides are increasingly being explored as alternative biofilm controls, and become important allies in the fight against bacterial tolerance and resistance $(28,29)$. As shown here, capsicumicine, a peptide derived from Capsicum baccatum red pepper seeds, possesses strong antibiofilm activity in vitro and in vivo. Capsicumicine prevents the establishment and maintenance of biofilm architecture through a new mechanism of action the "matrix anti-assembly" (MAA). MAA differs from matrix disassembly (30) as instead of de-structuring pre-established matrices it acts on the initial phase of assembly preventing its correct structuration. In fact, established biofilms are

294 harder to treat than initial biofilms, because they have more complex structures (31), and increased structural complexity means that more energy is required for disassembly (32).

Bacterial surface proteins can passively interact with surfaces such as medical devices, generating an initial and reversible adhesion after electrostatic and hydrophobic interactions, Van der Waals forces and others (33). Bacteria will then require extracellular matrix production in order to remain attached after these weak interactions (34). During this process, physicochemical interactions drive molecular and colloidal matrix self-assembly, establishing a chain of dense architecture that results in

301 stable adhesion (Fig. 6A) (35). In contrast, capsicumicine interacts with matrix saccharides and

302 modifies the self-assembly chain, resulting in a less dense and nonfunctional matrix, and impaired 303 biofilm formation (Fig. 6B).

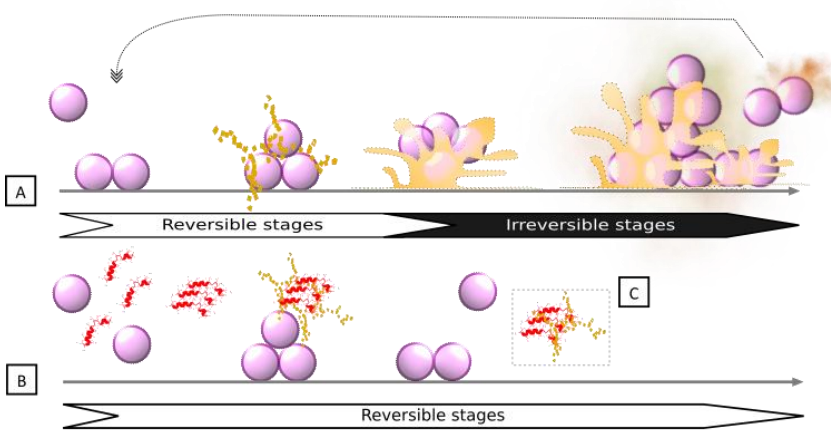

A $\sum \quad$ Reversible stage 2

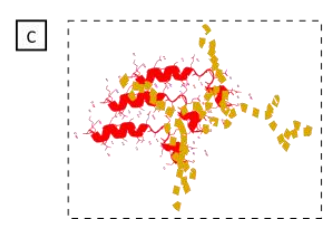


Fig. 6. The matrix anti-assembly (MAA), antibiofilm mechanism of action (MoA) identified in capsicumicine. (A) Untreated biofilm development on abiotic surfaces: planktonic cells interact passively with the surface and start the extracellular matrix production. Initial adhesion is established, after which matrix self-assembly occurs, leading to irreversible biofilm structuring and adhesion. (B) Biofilm development in the presence of capsicumicine shows the MAA MoA: planktonic cells are still able to interact passively with the surface. At that point, capsicumicine acts, attracting extracellular saccharides and shifting the matrix self-assembly; (C) Capsicumicine (red) tends to organize itself in beta leaves favoring the interactions with matrix target-saccharides (yellow). These interactions occur between the non-catalytic carbohydrate binding modules of the peptide and the saccharides. This peptide supra organization can compete with matrix assembly, preventing/destroying intra matrix h-bonds over a large range of residues. Consequently, the MAA decreases adhesion and aggregation, forcing bacteria to remain planktonic. The arrows indicate

Capsicumicine's amino acid sequence contains several residue characteristics for recognizing putative chitin-binding domains, including polar and hydrophobic residue ( $45 \%$ of $Q$ ) and cysteines (36-39). The chitin-binding domain is a well-conserved amino acid stretch that binds specifically to Nacetyl glucosamine, a homologous structure of PIA $(27,40,41)$. Additionally, non-catalytic carbohydrate binding modules (CBMs) are contiguous amino acid sequences with a discreet fold displaying carbohydrate-binding activity. In this context, capsicumicine displays homologies with all tested CBM-proteins, notably with icaA protein that is a PIA synthetase from the same S. epidermidis strain studied here (Fig. S3 and Table S2). secondary structures ranging from mainly $\beta$-sheet based organization to a mixture of $\alpha$-helices and $\beta$ sheets and usually display a high content of loops and unfolded parts. Likewise, capsicumicine secondary fold is a mixture of $\alpha$-helix, $\beta$-sheet and unfolded regions, with conformational time and

332 temperature interconversion in favor of $\beta$-sheet and unfolded segments. The $\beta$-sheet sub-structure

333 observed in the CD concerns the Nter part of the peptide according to the chemical shifts of the

334 corresponding amino acids (between 8.6 and 9.0). The TOCSY spectra analyses disclose that the

335 conformational changes observed through the time are originated around the two cysteines, possibly

336 involved in intermolecular disulfide bonds. This disulfide bond is possibly intermolecular since the 
337 formation of an intramolecular disulfide bond is only possible by bending the structure to get both

338 cysteines close to each other. The size of different conformers and potential polymers are limited as

339 shown by the NMR peaks of free peptides which are not broadened at day 7 (Fig. 4C). As a result,

340 these conformational changes may correspond to structural requirements toward to interactions

341 with staphylococcal matrix.

342 Antibiofilm, matrix anti-assembly (MAA) mechanism of action

343 This proposed mechanism is based on a set of intermolecular (i) and cooperative (ii) forces triggered

344 by capsicumicine which then disrupt matrix assembly:

\section{5 (i) Intermolecular forces}

346 The staphylococcal matrix is composed mainly of polysaccharides, but also contains proteins (AtIE,

347 Aap, Empb), teichoic acids, and extracellular DNA (42). These saccharides are PIA/PNAG,

348 homoglycans of beta-1,6-linked 2-amino-2-deoxy-D-glucopyranosyl residues. The matrix contains

349 positively-charged amino groups (PNAG), as well as negative charges from O-succinylation, which

350 confers electrical charge lability on the matrix.

351 Supported by NMR observations, capsicumicine interacts with free chitosan, a PNAG mimetic, and

352 then switches to a higher molecular weight organization (Fig 3B). The line broadening observed with

353 the peptide-chitosan mixture after one day evidence the aggregation of chitosan particles with

354 capsicumicine over the time as schematized on Fig 6. Likewise, CSPs analysis reveals that chitosan is

355 able to bind preferentially capsicumicine N-terminus (Fig $4 E$ ) before intermolecular disulfide bridges

356 formation and the relative conformational transition. This is supported by the fact that chitosan is

357 sub-stoichiometric at the end of the NMR titration and because the capsicumicine spectrum of the

358 mixture is different from the spectra of the free peptide (Fig $4 C$ ). These interactions may proceed by

359 both hydrogen-bonding between the hydrophilic Q's lateral chains and hydrophobic/stacking

360 interactions involving I and L residues. According to NMR, the N-terminal part of the peptide is 
361 probably the favored interacting region with the carbohydrate moieties. Thus, these data support

362 the MAA model in which the peptide supra organization would compete with matrix self-assembly

363 preventing intra h-bonds over a large range of residues. However, the NMR results obtained with

364 assembled chitosan, mimetizing a preformed biofilm, demonstrate that capsicumicine does not

365 interact with pre-assembled matrix $(\mathrm{Fig} 3 C, D)$ such as demonstrated in the eradication test.

366 Additionally, the amino acids in capsicumicine are mostly neutral, counting $13 \mathrm{Q}$ and $3 \mathrm{~S}$ although,

367 due to Q's amide group and S's hydroxyl group, they can generate electronegative (dipole-dipole)

368 zones. This high electronegativity suggests that capsicumicine may interact with the positive free

369 charges of the PIA/PNAG. Thus, these polar non-ionic forces perform moderate interactions with the

370 polysaccharides. Since strong interactions such as ionic forces may trigger unwanted effects including

371 matrix repulsion or sequestration $(43,44)$, the moderate interactions of capsicumicine seems to be

372 ideal.

373 (ii) Cooperative polymerization forces

374 In living systems, biomolecules perform their functions in the presence of various macromolecules of

375 different shapes and sizes, and these interactions can include cooperative polymerization forces such

376 as depletion forces (DFs) and subsequent molecular crowding (MC) $(45,46)$. These forces are

377 noncovalent and non-specific physicochemical interactions leading to bridging, aggregation, and

378 rheological variations $(47,48)$, as is observed in the presence of capsicumicine (Fig. $2 D-G$ and $3 A$ ),

379 and also may contribute to MAA. In a suspension containing different molecules, DFs are the

380 pressures exerted by small particles, which in turn cause attractive forces between the

381 macromolecules. DFs are only expressed in crowded environments like biofilms, driving the assembly

382 and final shape of these structures (49). Taking together NMR and CD experimental results as well as

383 the TANGO and SALSA analysis (Fig. S5B), we can picture that capsicumicine can self-organize to form

384 extended structures both by disulfide bridges and $\beta$-sheet mediated fibrils formation. We propose

385 that capsicumicine-associated DFs may coagulate in polymer solutions forming fibers and parallel 
bundles (50), explaining the observed branch-like profile (Fig. 2D-G) (51). This higher organization

387 should facilitate the interaction with the saccharides of the matrix preventing their pattern assembly

388 and resulting in flocculation (52). In a same way, MC in macromolecule solutions is characterized by

389 a decrease in accessible volume due to high macromolecule concentrations, as well as attractive and

390 repulsive forces between them $(45,53)$. Some molecular crowders modulate refolding kinetics and

391 decrease competing aggregation and segregation (54). In this way, capsicumicine enhances the

392 aggregation kinetics (Fig. 3A), acting as an agent of MC, decreasing entropic forces and leading to

393 segregation (55).

394 Thus, capsicumicine' structures are chemically enable to interact with staphylococcal matrices

395 saccharides. These combined intermolecular and cooperative forces disturb matrix self-assembly at

396 both molecular and colloidal levels. Consequently, matrix functionality shifts and antibiofilm activity

397 occurs. In other hand, planktonic microorganisms are more available for the innate immune system

398 to recognize and clear them (56). Furthermore, non-antibiotic activity appears less susceptible to the

399 development of bacteria resistance because these microorganisms are under less evolutionary

400 pressure then when exposed to conventional antibiotics $(57,58)$.

401 Finally, we demonstrated that capsicumicine strongly prevents biofilm formation for the most

402 frequent bacteria related to nosocomial infections, S. epidermidis and S. aureus. We first confirmed

403 that capsicumicine was not cytotoxic thus being safe for in vivo tests. Then, we trialed the peptide in

404 a translational pre-clinical model that mimics medical device-related infection. As a result, pre-

405 coated CVCs enhanced mice survival decreasing S. aureus colonization and consequently attenuated

406 the infection.

407 Although there are no antibiofilm drugs available here we relate the discovery of the first-in-class

408 carbohydrate-binding peptide as a promising candidate for complementary drug/treatment of

409 infectious diseases. In particular, we elucidated its antibiofilm mechanism of action, matrix anti-

410 assembly (MAA) and validated a proof of concept towards to an in vivo application. 
413 Peptides. All peptides were synthesized by Biomatik and ProteoGenix at purity grades over $95 \%$ in

414 salts suitable for cell culture. For the assays, the peptides were all solubilized in ultra-pure sterile

415 water.

416 Bacterial strains and growth conditions. Staphylococcus epidermidis ATCC 35984 was grown

417 overnight on blood agar (Thermo Scientific Oxoid PB5039A) at $37^{\circ} \mathrm{C}$. Oxoid LB agar was used for the

418 colony-forming unit (CFU) assay. The other assays were done using a bacterial suspension of $3 \times 10^{8}$

$419 \mathrm{CFU} / \mathrm{mL}$ in tryptone soya broth (TSB, Oxoid) or $0.9 \% \mathrm{NaCl}$.

420 Biofilm formation. At least three technical and biological replicates were done for each assay of 1 ,

421 10, or $100 \mu \mathrm{M}$ peptide concentrations. Biofilm formation inhibition: A protocol adapted from Trentin

422 et al (59) was used, with crystal violet in 96-well BD Falcon polyvinyl chloride (PVC) microtiter plates.

423 The cell-bound stains were solubilized with absolute ethanol (Sigma-Aldrich), and absorbance was

424 measured at $570 \mathrm{~nm}$ using a BIO-TEK PowerWave XS plate reader. The biofilm formation control

425 represents $100 \%$ of biofilm formation. Biofilm eradication: Biofilm was pre-formed as described

426 above for $24 \mathrm{~h}$ at $37^{\circ} \mathrm{C}$ without treatment. Afterwards, the wells were washed to remove planktonic

427 cells, peptide solutions and controls were added, and all were incubated for $24 \mathrm{~h}$. Biofilm eradication

428 was verified by evaluating the remaining content by crystal violet.

429 Bacterial growth assays. Microtiter plates: Bacterial growth was evaluated by comparing $\mathrm{OD}_{600}$

430 values at the start and end of incubation in 96-well PVC microtiter plates. Colony-forming units: After

431 incubation at $37^{\circ} \mathrm{C}$ for $24 \mathrm{~h}, \mathrm{CFU} / \mathrm{mL}$ was calculated to determine the peptide solution's bactericidal

432 effects. The untreated growth control was considered to be $100 \%$ planktonic cells. At least three

433 technical and biological replicates were performed for all assays. 
434 Quantitative reverse transcription PCR (qRT-PCR): After culturing for $24 \mathrm{~h}$, RNAs were isolated from

435 planktonic controls, biofilm controls, and from total cells exposed to $10 \mu \mathrm{M}$ capsicumicine. An

436 Invitrogen TRIzol Max bacterial RNA isolation kit and an Ambion TURBO DNase treatment were used

437 as per manufacturer instructions. Total RNA concentrations and purities were assessed using a

438 Biochrom SimpliNano spectrophotometer, and PCR reactions was done to ensure the complete

439 absence of DNA. Each qRT-PCR reaction was then subjected to previously established quantities of

440 cDNA (10 ng) and primers (0.2 $\mu \mathrm{M})$. Reactional volumes were calculated using SYBR Select Master

441 Mix (Applied Biosystems), as per the manufacturer's instructions. Primers (see Table S1) were

442 designed using the Primer3 program, then produced by Eurofins Genomics. Applied Biosystems

443 StepOnePlus equipment and software were used. Relative transcript levels were determined by the

$444 \quad 2^{-\Delta \Delta c t}$ method (60).

445 Cytotoxicity assays: Cytotoxicity assays were performed on the ImPACcell robotic platform (BIOSIT,

446 Université de Rennes 1). Multiparameter high-content screening (HCS) and high-content analysis

447 (HCA) were done on 7 different mammalian lines: HuH7, CaCo-2, MDA, HCT116, PC3, NCI-H727, and

448 MCF7. The number of normal cells is presented as residual cell percentage compared to the DMSO

449 control average.

450 Microscopic analysis. S. epidermidis ATCC 35984 biofilm was cultured as described above.

451 Scanning electron microscopy (SEM): Sterile 10x4mm polystyrene coupons were inserted into

452 bacterial cultures in the presence or absence of capsicumicine for 1,4 , and $24 \mathrm{~h}$. The coupons were 453 then washed with sterile $0.9 \% \mathrm{NaCl}$ and fixed with $2.5 \%$ glutaraldehyde, $2 \%$ paraformaldehyde, and $454 \quad 0.1 \mathrm{M}$ cacodylate buffer ( $\mathrm{pH}$ 7.2). Afterwards, they were washed with $0.1 \mathrm{M}$ cacodylate buffer and $4550.2 \mathrm{M}$ sucrose, then dehydrated with increasing concentrations of ethanol. A Leica EM CPD300 was 456 used for critical point drying of the dehydrated samples. These were then sputtered with palladium 457 in a Leica EM ACE200, and analyzed with a JEOL JSM-7100F microscope with EDS and EBSD at $10 \mathrm{kV}$. 
458 Transmission electron microscopy (TEM): All well content was carefully detached at 1, 4, and $24 \mathrm{~h}$,

459 centrifuged at $10,000 \mathrm{~g}$ for $15 \mathrm{~min}$ at $4{ }^{\circ} \mathrm{C}$, then washed with sterile $0.9 \% \mathrm{NaCl}$. Fixation was

460 performed at $4{ }^{\circ} \mathrm{C}$ with sodium $0.1 \mathrm{M}$ cacodylate, $2 \%$ paraformaldehyde, $2.5 \%$ glutaraldehyde, and 75

$461 \mathrm{mM}$ lysine. Samples were washed with $0.1 \mathrm{M}$ sodium cacodylate and $0.2 \mathrm{M}$ sucrose and contrasted

462 with $1 \%$ osmium tetroxide and $1.5 \%$ potassium ferrocyanide. Dehydration was done with a gradual

463 solution of ethanol and infiltration of increasing concentrations of LR White resin (Delta

464 Microscopies, France). LR White resin inclusion and polymerization were then performed over $24 \mathrm{~h}$

465 at $60{ }^{\circ} \mathrm{C}$ in the absence of $\mathrm{O}_{2}$. Thin $80 \mathrm{~nm}$ sections were collected onto carbon grids, and visualized at

466200 kV with a FEI Tecnai Sphera microscope equipped with a Gatan 4k x 4k CCD UltraScan camera.

467 Confocal fluorescence microscopy (CFM): Capsicumicine-fluorescein isothiocyanate (capsicumicine-

468 ITC, $10 \mu \mathrm{M}$ ) was used to detect the capsicumicine peptide, whose antibiofilm activity was previously

469 verified. After incubation for 1,4 , or 24 h, the well contents were carefully detached, centrifuged at

$47011,000 \mathrm{~g}$ for $2 \mathrm{~min}$ at $4{ }^{\circ} \mathrm{C}$, then washed with sterile $0.9 \% \mathrm{NaCl}$. The suspension was visualized directly

471 or after adding $0.1 \mu \mathrm{g} / \mu \mathrm{L}$ concanavalin A conjugates (Alexa Fluor ${ }^{\circledR} 633$, Invitrogen) or $2 \mathrm{mg} / \mathrm{mL}$

472 Calcofluor White dye (Fluorescent Brightener 28, Sigma-Aldrich). To find bacterial cells permeated by

473 the peptide control, we used pseudonajide FITC-labelled antimicrobial peptide (Ref). Images were

474 acquired via resonant scanner with a Leica SP8 DMI 6000 CS confocal microscope with hybrid

475 detector, and ImageJ software was used for image analysis.

476 Real-time molecular self-assembly (RTMSA) assay: After checking the starting point (pH 7.2) of the

477 assembly reaction for the synthetic staphylococcal matrix (35), we recorded the $\mathrm{OD}_{600}$ as a function

478 of the time every $30 \mathrm{sec}$ until $30 \mathrm{~min}$. Molecular self-assembly reactions were calculated to a final

479 volume of $4 \mathrm{~mL}$, with $0.3 \%$ chitosan (medium molecular weight, $75-85 \%$ deacetylation), $0.15 \%$ bovine

480 serum albumin, and $0.015 \%$ lambda DNA (all from Sigma) in TSB. The concentration ( $\mu \mathrm{M}$ ) of tested

481 peptides was calculated for a final volume of $4 \mathrm{~mL}$. Before getting the assembly reaction pH starting

482 points, a calibration record was done using the same reactional tube containing all reagents (auto 
zero). Acetic acid and $\mathrm{NaOH}$ were used to adjust $\mathrm{pH}$, and the reaction temperature was about $30^{\circ} \mathrm{C}$.

484 As a negative control a similarly sized peptide was used, PA-1 (61).

NMR: NMR spectra were recorded in 3mm tubes on a Bruker Avance III 600MHz spectrometer equipped with a TXI $(1 \mathrm{H}, 13 \mathrm{C}, 15 \mathrm{~N})$ probe and a Z-gradient unit. Spectra were processed with Topspin 4.0.8 (Bruker Biospin) and CcpNmr Analysis (62). TOCSY and NOESY experiments were respectively recorded at $10^{\circ} \mathrm{C}$ and $\mathrm{pH} 5.0$ with a $70 \mathrm{~ms}$ and $300 \mathrm{~ms}$ mixing time. Capsicumicine concentration in water was set at $0.3 \mathrm{mM}$ and the $\mathrm{pH}$ adjusted either 5.0 or to 3.5 with few microliters of deuterated $\mathrm{HCl}$ 0.1M and/or $\mathrm{NaOH} 0.1 \mathrm{M}$ solutions. Chitosan powder (purchased from Sigma) was dissolved in 491 pure water and the $\mathrm{pH}$ adjusted to either 5.0 or 3.5. Reference 1D proton NMR spectra were 492 recorded at $10^{\circ} \mathrm{C}$ and either at $\mathrm{pH} 3.5$ and 5.0. Chitosan was stepwise added from a concentrated 493 solution at $\mathrm{pH} 5.0$ or 3.5 and spectra changes were monitored. We first titrated capsicumicine solution with chitosan solution at pH 5.0. The concentration variation of chitosan was estimated using the peaks intensities, resulting in 3.6 and $3.8 \mathrm{ppm}$ (not shown) in the beginning to less than $0.5 \mathrm{eq}$ at the end of the titration. These experiments were repeated with a starting capsicumicine

497 solution at $\mathrm{pH} 3.5$ and the gradually addition of gelled chitosan, at $\mathrm{pH}$ 3.5. The suddenly addition of 498 gelled chitosan pellets in the NMR solution, at pH3.5.

499 Circular Dichroism (CD): The peptide was dissolved in 18Ma water at a concentration of $50 \mu \mathrm{M}$, at pH 500 5.0. The ultraviolet $C D$ spectra were recorded at 5,15 and $30^{\circ} \mathrm{C}$, in a $0.1 \mathrm{~cm}$ path length quartz cell on 501 a Jobin Yvon CD6 spectrometer equipped with a temperature controller unit, over a 180-260 nm 502 range, with a $2 \mathrm{~nm}$ bandwidth, a step size of $1 \mathrm{~nm}$ and an integration time of $2 \mathrm{~s}$ per point. The 503 samples were conserved at $5^{\circ} \mathrm{C}$ between each recording. Spectra were averaged over 5 records.

504 Water CD contributions were subtracted from CD spectra before processing. Spectra were processed 505 using Kaleidagraph (Synergy Software). Molar circular dichroism (ㅁ]물 per residue and molar ellipticity 506 per residue (ㅁ?] ], MER) were computed from the difference of the delta absorbance recorded by the 507 spectrometer. Raw delta epsilon per residue spectra were analyzed using the CDSSTR program (63) 
and different reference protein data sets provided by the DICHROWEB facility (64). MER data curves

509 were smoothed for presentation, using the interpolate and weighted data (5\%) routines provide by

510 Kaleidagraph.

511 TANGO (65) and SALSA (66) predictions: Tango predictions were run at 298K. Predictions were

512 identical for $\mathrm{pH}$ set either to $5.0,7.0$ or 9.0. SALSA predictions were run with a window size dynamic 513 of 4-20 residues, a cutoff of 1.2 and a minimal hot spot length of 5.

514 CVC coatings: We adapted an approach to immobilize peptides based on poly(ethylene

515 glycol)diacrylate (PEGDA) hydrogel (67). Briefly, polyurethane (PU) tubes (Instech) of 2Fr or

$51625 \mathrm{G}$ equivalent were used as coating framework. Hydrogel base was prepared using

517 pentaerythritol tetrakis(3-mercaptopropionate) - PTMP (4.1 mmol), PEGDA (10 mmol), PEG-

518600 (20mM), 2,2-Dimethoxy-2-phenylacetophenone - DMPA (0.1 wt\%), THF soluble PU (10

519 wt\%) and methanol (qs). Peptides were solubilized in DMSO and added to hydrogel base

520 under vortex agitation. PU tubes were first internally coated by suction using a needle-

521 syringe and then externally by immersion. The reaction and polymerization conditions such

522 as room temperature and oxygen tolerance were convenient. After polymerization,

523 successive washes with methanol under agitation were used to eliminate undesirable

524 monomers. All reagents were purchased from Sigma Aldrich.

525 CVC infection assay: This study was conducted by Voxcan s.a.r.I. The ethical agreement is part of the

526 project $n^{\circ}$ APAFiS\# 10756-2017072522272676 v4, approved by Voxcan ethical committee (CEAA-129)

527 and the French authorities (ministry of national education, the higher education and research). This

528 study used SKH1 mice, females, immunocompetent and specific pathogen free (SPF) provided by

529 Charles River Laboratories. Animals were acclimated at least 2 days, housed collectively in disposable

530 standard cages in ventilated racks $A 3$, at $+21 \pm 3^{\circ} \mathrm{C}, 30-70 \%$ of humidity, 12 hours of dark and light

531 cycles, with filtered water and autoclaved standard food provided ad libitum. Catheters were blind 
532 implanted ( $n=5$ / group) in mice jugular vein following by an intravenous (IV) inoculation of $S$.

533 aureus Xen36, 5 days days later, which was in turn to colonize the device from the blood circulation.

534 The bacterial development was evaluated and compared between the different catheters by in vivo

535 bioluminescence imaging (IVIS Spectrum, acquisition and analysis with Living Image 4.5.5 version)

536 performed 2 and 4 days after mice infection. In addition, 7 days after inoculation or at the time of

537 mice euthanasia for ethical reason, catheters were harvested and bacterial load were evaluated by

538 CFU counting (SCAN500). All along the study, mice clinical state was evaluated using a scoring grid

539 and body weight measurements 3 times a week. At each timepoint, a bioluminescence acquisition

540 was also performed on the background (BKG) mouse to measure the flux level corresponding to the

541 auto-bioluminescence. Images were radiance-thresholded with respect to the background radiance

542 level and smoothed with a bidimensional Gaussian filter (3×3).

\section{Acknowledgments:}

545 General: We thank Daniel Thomas for his support, and Juliana Berland for insightful comments on

546 the manuscript. Thanks to the Microscopy Rennes Imaging Center (MRic) and CMEBA facilities.

547 Funding: This study was funded by the CAPES-COFECUB program, whose institutional partners are

548 the Brazilian Ministry of Education's CAPES (Coordenação de Aperfeiçoamento de Pessoal de Nível

549 Superior) agency and the French Ministère de l'Europe et des Affaires Étrangères (MEAE) and

550 Ministère de l'Enseignement supérieur, de la Recherche et de l'Innovation (MESRI). Support was also

551 received from the Transfer Acceleration Company SATT Ouest-Valorisation. Author contributions:

552 R.G.V.B, S.C.B.G, A.J.M, S.N-L. and R.G. designed research; R.G.V.B, S.C., R.S., S.N-L. and S.B.

553 performed research; R.G.V.B, A.R.Z., E.G., S.C.B.G, A.J.M, S.N-L., S.B. and R.G. analyzed data; and

554 R.G.V.B, A.J.M, S.N-L. and R.G. wrote the paper. Competing interests: R.G.V.B, S.C.B.G, A.R.Z, A.J.M

555 and R.G. are co-authors of a patent register of capsicumicine. Application number WO 2020/169709 
A1 at European Patent Office. Specific aspect of manuscript covered in patent application: its amino acid sequence and bioactivity.

Data and materials availability: All data needed to evaluate the conclusions in the paper are present requested from the authors.

\section{References:}

563 1. WHO, W. H. Organization. (2015).

564 2. WHO, W. H. Organization. (2018).

565 3. A. Penesyan, M. Gillings, I. T. Paulsen, Antibiotic discovery: combatting bacterial resistance in cells and in biofilm communities. Molecules 20, 5286-5298 (2015).

4. H.-C. Flemming, J. Wingender, The biofilm matrix. Nature Reviews Microbiology 8, 623-633 (2010).

5. A. Taglialegna et al., Staphylococcal Bap Proteins Build Amyloid Scaffold Biofilm Matrices in Response to Environmental Signals. PLoS Pathog 12, e1005711 (2016).

6. N. Hoiby, T. Bjarnsholt, M. Givskov, S. Molin, O. Ciofu, Antibiotic resistance of bacterial biofilms. International Journal of Antimicrobial Agents 35, 322-332 (2010).

7. C. Beloin, S. Renard, J. M. Ghigo, D. Lebeaux, Novel approaches to combat bacterial biofilms. Curr Opin Pharmacol 18C, 61-68 (2014).

8. D. Davies, Understanding biofilm resistance to antibacterial agents. Nat Rev Drug Discov 2, $114-122(2003)$.

9. A. Brauner, O. Fridman, O. Gefen, N. Q. Balaban, Distinguishing between resistance, tolerance and persistence to antibiotic treatment. Nat Rev Microbiol 14, 320-330 (2016).

10. V. Defraine, M. Fauvart, J. Michiels, Fighting bacterial persistence: Current and emerging anti-persister strategies and therapeutics. Drug Resist Updat 38, 12-26 (2018).

11. M. Otto, Physical stress and bacterial colonization. FEMS Microbiol Rev 38, 1250-1270 (2014).

12. I. Uçkay et al., Meticillin resistance in orthopaedic coagulase-negative staphylococcal infections. J Hosp Infect 79, 248-253 (2011).

13. M. Otto, Staphylococcal biofilms. Bacterial Biofilms 322, 207-228 (2008).

14. Y. Nishizaki et al., Japanese features of native valve endocarditis caused by coagulasenegative staphylococci: case reports and a literature review. Intern Med 52, 567-572 (2013).

15. C. L. Abad, A. Haleem, Prosthetic Joint Infections: an Update. Curr Infect Dis Rep 20, 15 (2018).

16. M. E. Rupp, Clinical characteristics of infections in humans due to Staphylococcus epidermidis. Methods Mol Biol 1106, 1-16 (2014).

17. $\mathrm{CDCP}$, Center for Disease Control and Prevention. Vital signs: central line-associated blood stream infections -United States, 2001, 2008, and 2009. MMWR Morb Mortal Wkly Rep 60, 243-248 (2011).

18. J. Y. H. Lee et al., Global spread of three multidrug-resistant lineages of Staphylococcus epidermidis. Nat Microbiol, (2018). 
19. J. M. Streit, R. N. Jones, H. S. Sader, T. R. Fritsche, Assessment of pathogen occurrences and resistance profiles among infected patients in the intensive care unit: report from the SENTRY Antimicrobial Surveillance Program (North America, 2001). Int J Antimicrob Agents 24, 111-118 (2004).

20. R. K. Flamm et al., Linezolid Surveillance Results for the United States (LEADER Surveillance Program 2014). Antimicrob Agents Chemother 60, 2273-2280 (2016).

21. R. Hope et al., Non-susceptibility trends among staphylococci from bacteraemias in the UK and Ireland, 2001-06. J Antimicrob Chemother 62 Suppl 2, ii65-74 (2008).

22. N. Strempel, J. Strehmel, J. Overhage, Potential Application of Antimicrobial Peptides in the Treatment of Bacterial Biofilm Infections. Current Pharmaceutical Design 21, 67-84 (2015).

23. C. de la Fuente-Nunez et al., D-Enantiomeric Peptides that Eradicate Wild-Type and Multidrug-Resistant Biofilms and Protect against Lethal Pseudomonas aeruginosa Infections (vol 22, pg 196, 2015). Chemistry \& Biology 22, 1280-1282 (2015).

24. O. M. El-Halfawy et al., Discovery of an antivirulence compound that reverses $\beta$-lactam resistance in MRSA. Nat Chem Biol 16, 143-149 (2020).

25. R. G. V. Borowski et al., Red pepper peptide coatings control Staphylococcus epidermidis adhesion and biofilm formation. Int J Pharm 574, 118872 (2020).

26. The UniProt Consortium, UniProt: the universal protein knowledgebase. Nucleic Acids Res 45, D158-D169 (2017).

27. V. Lombard, H. Golaconda Ramulu, E. Drula, P. M. Coutinho, B. Henrissat, The carbohydrateactive enzymes database (CAZy) in 2013. Nucleic Acids Res 42, D490-495 (2014).

28. C. Feuillie et al., Molecular interactions and inhibition of the staphylococcal biofilm-forming protein SdrC. Proc Natl Acad Sci U S A 114, 3738-3743 (2017).

29. S. Schmitt et al., Analysis of modular bioengineered antimicrobial lanthipeptides at nanoliter scale. Nat Chem Biol 15, 437-443 (2019).

30. R. Roy, M. Tiwari, G. Donelli, V. Tiwari, Strategies for combating bacterial biofilms: A focus on anti-biofilm agents and their mechanisms of action. Virulence 9, 522-554 (2018).

31. S. Jabbouri, I. Sadovskaya, Characteristics of the biofilm matrix and its role as a possible target for the detection and eradication of Staphylococcus epidermidis associated with medical implant infections. FEMS Immunol Med Microbiol 59, 280-291 (2010).

32. D. Fleming, K. P. Rumbaugh, Approaches to Dispersing Medical Biofilms. Microorganisms 5, (2017).

33. C. R. Armbruster, M. R. Parsek, New insight into the early stages of biofilm formation. Proc Natl Acad Sci U S A 115, 4317-4319 (2018).

34. M. Otto, Staphylococcal Infections: Mechanisms of Biofilm Maturation and Detachment as Critical Determinants of Pathogenicity. Annual Review of Medicine, Vol 64 64, 175-188 (2013).

35. E. J. Stewart, M. Ganesan, J. G. Younger, M. J. Solomon, Artificial biofilms establish the role of matrix interactions in staphylococcal biofilm assembly and disassembly. Sci Rep 5, 13081 (2015).

36. H. Hemmi, J. Ishibashi, T. Tomie, M. Yamakawa, Structural basis for new pattern of conserved amino acid residues related to chitin-binding in the antifungal peptide from the coconut rhinoceros beetle Oryctes rhinoceros. J Biol Chem 278, 22820-22827 (2003).

37. T. Suetake et al., Chitin-binding proteins in invertebrates and plants comprise a common chitin-binding structural motif. J Biol Chem 275, 17929-17932 (2000).

38. H. T. Wright, G. Sandrasegaram, C. S. Wright, Evolution of a family of N-acetylglucosamine binding proteins containing the disulfide-rich domain of wheat germ agglutinin. $J$ Mol Evol 33, 283-294 (1991).

39. J. Yin, S. Yang, K. Li, W. Guo, Y. Cao, Identification and molecular characterization of a chitinbinding protein from the beet webworm, Loxostege sticticalis L. Int J Mol Sci 15, 1914719161 (2014). 
40. S. El-Gebali et al., The Pfam protein families database in 2019. Nucleic Acids Res 47, D427-

648

649

650

651

652

653

654

655

656

657

658

659

660

661

662

663

664

665

666

667

668

669

670

671

672

673

674

675

676

677

678

679

680

681

682

683

684

685

686

687

688

689

690

691

692

693

694

695

696

697

D432 (2019).

41. W. Suginta, P. Sirimontree, N. Sritho, T. Ohnuma, T. Fukamizo, The chitin-binding domain of a GH-18 chitinase from Vibrio harveyi is crucial for chitin-chitinase interactions. Int J Biol Macromol 93, 1111-1117 (2016).

42. A. E. Paharik, A. R. Horswill, The Staphylococcal Biofilm: Adhesins, Regulation, and Host Response. Microbiol Spectr 4, (2016).

43. G. Batoni, G. Maisetta, S. Esin, Antimicrobial peptides and their interaction with biofilms of medically relevant bacteria. Biochimica Et Biophysica Acta-Biomembranes 1858, 1044-1060 (2016).

44. A. Sharma, P. Gupta, R. Kumar, A. Bhardwaj, dPABBs: A Novel in silico Approach for Predicting and Designing Anti-biofilm Peptides. Sci Rep 6, 21839 (2016).

45. S. F. Banani, H. O. Lee, A. A. Hyman, M. K. Rosen, Biomolecular condensates: organizers of cellular biochemistry. Nat Rev Mol Cell Biol 18, 285-298 (2017).

46. C. Rest, R. Kandanelli, G. Fernández, Strategies to create hierarchical self-assembled structures via cooperative non-covalent interactions. Chem Soc Rev 44, 2543-2572 (2015).

47. A. Kudlay, M. S. Cheung, D. Thirumalai, Influence of the shape of crowding particles on the structural transitions in a polymer. J Phys Chem B 116, 8513-8522 (2012).

48. C. Even et al., Recent advances in studying single bacteria and biofilm mechanics. Adv Colloid Interface Sci 247, 573-588 (2017).

49. D. Marenduzzo, K. Finan, P. R. Cook, The depletion attraction: an underappreciated force driving cellular organization. J Cell Biol 175, 681-686 (2006).

50. H. X. Zhou, Effect of mixed macromolecular crowding agents on protein folding. Proteins 72, 1109-1113 (2008).

51. A. Sakaguchi, K. Higashiguchi, K. Matsuda, Bundle formation of supramolecular fibers of amphiphilic diarylethene by depletion force. Chem Commun (Camb) 54, 4298-4301 (2018).

52. R. Fantoni, A. Santos, Depletion force in the infinite-dilution limit in a solvent of nonadditive hard spheres. J Chem Phys 140, 244513 (2014).

53. W. M. Aumiller, B. W. Davis, C. D. Keating, Phase separation as a possible means of nuclear compartmentalization. Int Rev Cell Mol Biol 307, 109-149 (2014).

54. S. Biswas, J. Kundu, S. K. Mukherjee, P. K. Chowdhury, Mixed Macromolecular Crowding: A Protein and Solvent Perspective. ACS Omega 3, 4316-4330 (2018).

55. J. M. Polson, D. R. Kerry, Segregation of polymers under cylindrical confinement: effects of polymer topology and crowding. Soft Matter 14, 6360-6373 (2018).

56. C. de la Fuente-Núñez, F. Reffuveille, L. Fernández, R. E. Hancock, Bacterial biofilm development as a multicellular adaptation: antibiotic resistance and new therapeutic strategies. Curr Opin Microbiol 16, 580-589 (2013).

57. A. M. Krachler, K. Orth, Targeting the bacteria-host interface: strategies in anti-adhesion therapy. Virulence 4, 284-294 (2013).

58. L. Travier, O. Rendueles, L. Ferrieres, J.-M. Herry, J.-M. Ghigo, Escherichia coli Resistance to Nonbiocidal Antibiofilm Polysaccharides Is Rare and Mediated by Multiple Mutations Leading to Surface Physicochemical Modifications. Antimicrobial Agents and Chemotherapy 57, 39603968 (2013).

59. D. S. Trentin et al., Natural Green Coating Inhibits Adhesion of Clinically Important Bacteria. Scientific Reports 5, (2015).

60. K. J. Livak, T. D. Schmittgen, Analysis of relative gene expression data using real-time quantitative PCR and the 2(-Delta Delta C(T)) Method. Methods 25, 402-408 (2001).

61. P. Liu et al., Targeting Inhibition of SmpB by Peptide Aptamer Attenuates the Virulence to Protect Zebrafish against. Front Microbiol 8, 1766 (2017).

62. S. P. Skinner et al., CcpNmr AnalysisAssign: a flexible platform for integrated NMR analysis. $J$ Biomol NMR 66, 111-124 (2016). 
63. N. Sreerama, R. W. Woody, Estimation of protein secondary structure from circular dichroism spectra: comparison of CONTIN, SELCON, and CDSSTR methods with an expanded reference set. Anal Biochem 287, 252-260 (2000).

64. L. Whitmore, B. A. Wallace, DICHROWEB, an online server for protein secondary structure analyses from circular dichroism spectroscopic data. Nucleic Acids Res 32, W668-673 (2004).

65. A. M. Fernandez-Escamilla, F. Rousseau, J. Schymkowitz, L. Serrano, Prediction of sequencedependent and mutational effects on the aggregation of peptides and proteins. Nat Biotechnol 22, 1302-1306 (2004).

66. S. Zibaee, O. S. Makin, M. Goedert, L. C. Serpell, A simple algorithm locates beta-strands in the amyloid fibril core of alpha-synuclein, Abeta, and tau using the amino acid sequence alone. Protein Sci 16, 906-918 (2007).

67. R. T. C. Cleophas et al., Convenient Preparation of Bactericidal Hydrogels by Covalent Attachment of Stabilized Antimicrobial Peptides Using Thiol-ene Click Chemistry. ACS Macro Letters 3, 477-480 (2014).

68. L. A. Kelley, S. Mezulis, C. M. Yates, M. N. Wass, M. J. Sternberg, The Phyre2 web portal for protein modeling, prediction and analysis. Nat Protoc 10, 845-858 (2015).

Table S1. Primers used in this study. These were previously designed using the Primer3 program and standardized by our team.

\begin{tabular}{|c|c|c|c|}
\hline Gene & Forward primer 5'-3' & Reverse primer 5'-3' & Amplicon \\
\hline at/E & TACCAGGGTTTGGAGGATTC & GGCGCTAAATTCATTGGAAA & $85 \mathrm{pb}$ \\
\hline aap & AGGCCGTACCAACAGTGAAT & ATGGGCAAACGTAGACAAGG & $100 \mathrm{pb}$ \\
\hline $\operatorname{agrC}$ & TCATCAATATCGCATTCATCG & CCTAAACCGCGATTATCACC & $136 \mathrm{pb}$ \\
\hline$i c a A$ & TTATCAATGCCGCAGTTGTC & CCGTTGGATATTGCCTCTGT & $104 \mathrm{pb}$ \\
\hline leuA & GATGATCTCGGAATGGCAGT & TGAGGCATTTCCTGCTCTTT & $108 \mathrm{pb}$ \\
\hline saeR & GCTAACACTGTCAATGTCCACA & AGGCCCCACACAGTTGTAAT & $92 \mathrm{pb}$ \\
\hline saes & GGCGTCAATTTGTTGTGCTA & AGGGCATAGGTATCGTTCCA & $140 \mathrm{pb}$ \\
\hline sarA & TTTGCTTCTGTGATACGGTTGT & CGTAATGAACACGATGAAAGAACT & $107 \mathrm{pb}$ \\
\hline $\operatorname{gyr} B$ & ATCAACATCGGCATCAGTCA & GCATTTGGTACGGGTATTGG & $87 \mathrm{pb}$ \\
\hline $\operatorname{rrs} A$ & AAGCAACGCGAAGAACCTTA & ATGCACCACCTGTCACTCTG & $95 \mathrm{pb}$ \\
\hline
\end{tabular}


Table S2. Carbohydrate-binding domain proteins that are capsicumicine homologs. These proteins are available in the UniProtKB database and were used for BLAST and amino acid alignments to 722 perform similarity analysis.

723

724

725

726

727

\begin{tabular}{lll}
\hline Entry & Name & Accession ID \\
\hline \hline ICAA_STAEQ & Intercellular adhesion protein icaA & Q5HKQ0.1 \\
ICAD_STAEQ & Poly-beta-1,6-N-acetyl-D-glucos. synthase prot. icaD & Q5HKP9 \\
A7Z8H9_BACVZ & Chitosanase & A7Z8H9 \\
A0A0NOMLT5_9ACTN & Chitosanase & AOAONOMLT5 \\
CBP2_MOROL & Chitin-binding protein 2 & COHKC5 \\
A0A1R0GTZ5_9FUNG & Chitin synthase 8 & A0A1R0GTZ5 \\
A0A194V113_9PEZI & Chitin biosynthesis protein CHS5 & A0A194V113 \\
\hline
\end{tabular}

Table S3. Results of the CD spectra deconvolution using CDSSTR algorithm.

\begin{tabular}{|c|c|c|c|c|c|}
\hline Day & Temp $\left({ }^{\circ} \mathbf{C}\right)$ & $\begin{array}{c}\text { Helix } \\
(\%)\end{array}$ & $\begin{array}{c}\text { Bêta } \\
(\%)\end{array}$ & $\begin{array}{c}\text { Turn } \\
(\%)\end{array}$ & $\begin{array}{c}\text { Unfolded } \\
(\%)\end{array}$ \\
\hline 0 & 5 & $42-46$ & $29-34$ & $5-8$ & $16-21$ \\
\hline 1 & 5 & $42-45$ & $30-32$ & $6-8$ & $20-21$ \\
\hline 5 & 5 & $38-42$ & $24-31$ & $8-12$ & $17-23$ \\
\hline 5 & 15 & $22-26$ & $36-45$ & $13-14$ & $20-26$ \\
\hline 5 & 30 & $16-24$ & $33-40$ & $18-20$ & $20-25$ \\
\hline 7 & 30 & $6-17$ & $38-41$ & $10-23$ & $31-35$ \\
\hline
\end{tabular}

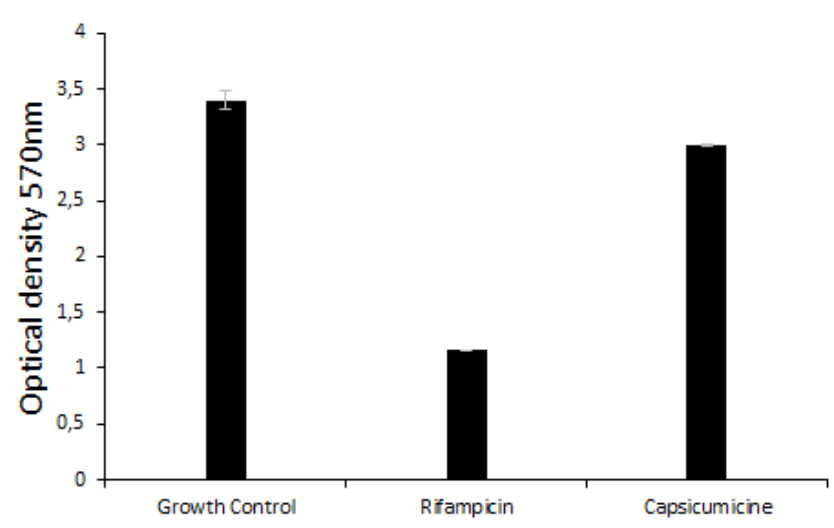

Fig. S1. Biofilm eradication test. Shown are Staphylococcus epidermidis (ATCC 35984) biofilm quantifications at $\mathrm{OD}_{570}$ for the bacterial biofilm without peptide exposure ("Growth Control"), after exposure to the rifampicin antibiotic control, and after $24 \mathrm{~h}$ treatment with $100 \mu \mathrm{M}$ capsicumicine. 

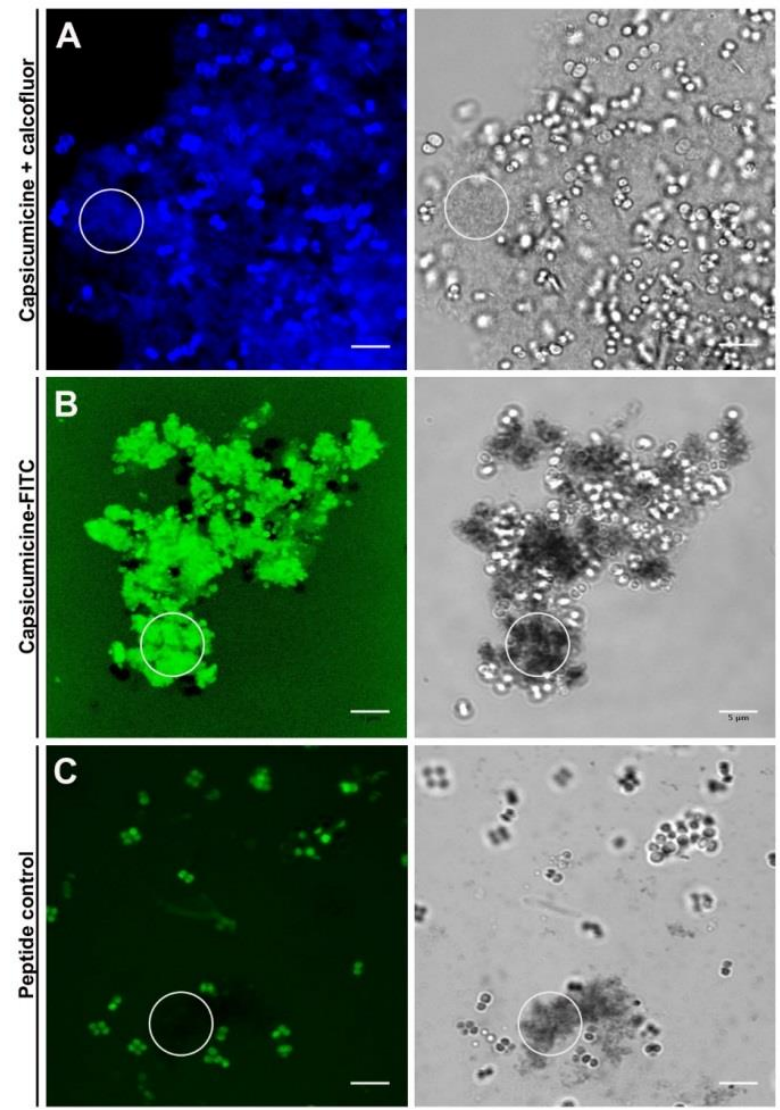

Fig. S2. Confocal fluorescence microscopy (CFM) of Staphylococcus epidermidis (ATCC 35984). Calcofluor was used to highlight matrix polysaccharides (blue), and FITC used for the peptides (green). Visualization was done by fluorescence (left) and transmitted light (right) microscopy. $(A)$ Cultures exposed to capsicumicine and calcofluor. (B) Cultures exposed to capsicumicine-FITC. (C) Peptide negative control cultures exposed to an antimicrobial peptide-FITC (Pseudonajide). The matrix is highlighted (circles). Scale bars, $5 \mu \mathrm{m}$. 


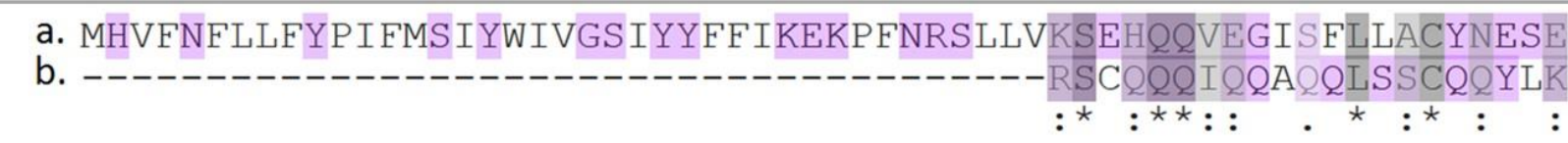

c. MKISLKKKAGFWKKTAVSSLIFTMFFTLMMSGTVFAAGLNKDQKRRAEQLTSIFENGKTE

d.

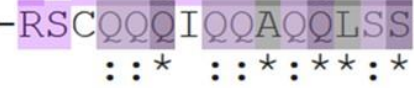

c. IQYGYVEALDDGRGYTCGRAGFTTATGDALEVVEVYTKAVPNNKLKKYLPELRRLAKDES

d. $: \star \star *:$

e. GNIHPGEYPNGGIYEHPRSVVSPLGFGRMYPNPEILYNPTPVNVHQLSLQQQIQQQQQQL

f. continuation

YAQAFSQQQIQQQYPQHQLYQHHLQQHQHQQHQLQQHHLQQHQLQQHQFQQQQLHQHHQQ $\mathrm{S}$ continuation

e. QINQQYQSQQHQNPSFQEMQLRQLNPVGSHQSQNVSSDAGIHHKPKD

f. $-\mathrm{CQQY}_{\star \star \star} \mathrm{LKQ}, \ldots$

742 Fig. S3. Amino acid alignment of capsicumicine and its carbohydrate-binding domain protein homologs. (a) The icaA (protein accession number Q5HKQ0.1) fragment from 1 to 60 . (b) Capsicumicine fragment from 1 to 21. (c) Chitosanase (A7Z8H9) fragment from 1 to 60 and from 61 to 120. (d) Capsicumicine fragment from 1 to 15 and from 16 to 22. (e) Chitin synthase 8 (AOA1R0GTZ5) fragment from 1801 to 1860 and from 1921 to 1967. (f) Capsicumicine fragment from 1 to 14 and from 16 to 22 . Equal (* and grey highlighting), similar (.), and highly similar (:) amino acids are indicated, as well as amino acid polar characteristics (purple). Support for this analysis is available at UniProt. 


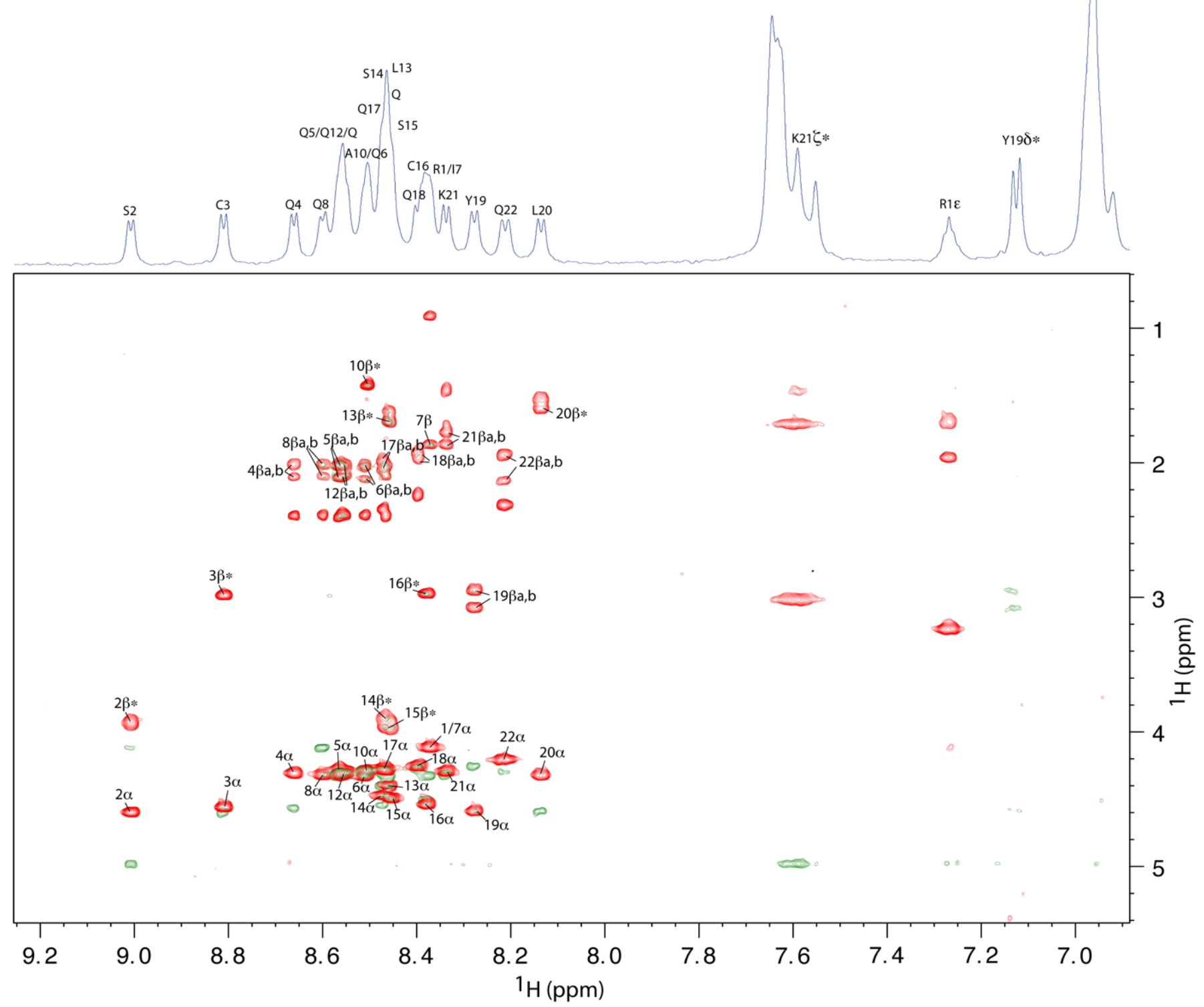

752 Fig. S4. Superposition of the TOCSY (red), NOESY (green) and 1D (blue) spectra of capsicumicine 753 recorded at $\mathrm{pH} 5.0$ and $10^{\circ} \mathrm{C}$. All amide $\mathrm{NH}$ resonances were assigned using $\mathrm{nNH}-(\mathrm{n}-1) \mathrm{H \alpha} \mathrm{NOESY}$ 754 cross-peaks, except for Q9 and Q11 residues due to poor resolution. For clarity, only NH-Ha and NH$755 \mathrm{H} \beta$ labels were reported. The specta display 22 spin systems as expected for a single conformation. 756 According to the spectral dispersion, capsicumicine is mainly folded. 
A

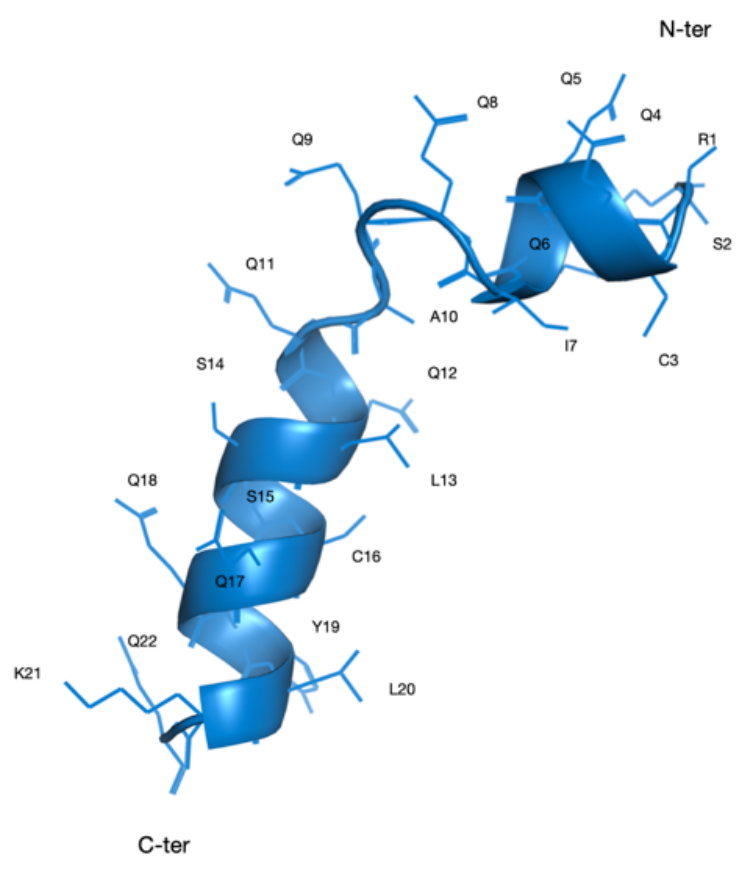

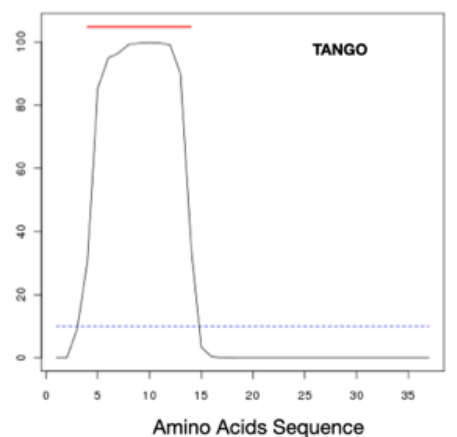

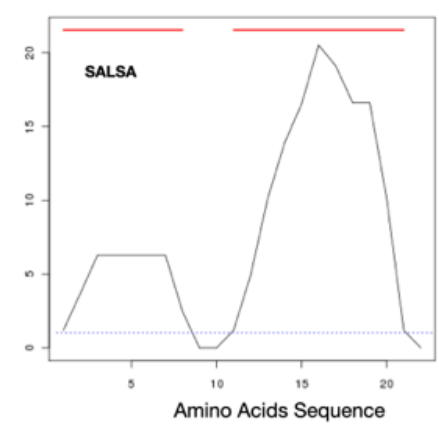

Results of aggregation predictions

\begin{tabular}{|c|c|c|c|}
\hline Position & Residue & TANGO & SALSA \\
\hline 1 & R & 0.0000 & 1.2020 \\
\hline 2 & S & 0.0000 & 3.7070 \\
\hline 3 & $\mathrm{C}$ & 9.0330 & 6.2770 \\
\hline 4 & $\mathrm{Q}$ & 30.2200 & 6.2770 \\
\hline 5 & $\mathrm{Q}$ & 85.3950 & 6.2770 \\
\hline 6 & $\mathrm{Q}$ & 94.9340 & 6.2770 \\
\hline 7 & $\mathrm{I}$ & 96.5820 & 6.2770 \\
\hline 8 & $\mathrm{Q}$ & 99.2880 & 2.4720 \\
\hline 9 & $\mathrm{Q}$ & 99.7080 & 0.0000 \\
\hline 10 & $\mathrm{~A}$ & 99.8980 & 0.0000 \\
\hline 11 & $\mathrm{Q}$ & 99.7820 & 1.2010 \\
\hline 12 & $\mathrm{Q}$ & 99.0890 & 4.9220 \\
\hline 13 & $\mathrm{~L}$ & 89.8320 & 10.0960 \\
\hline 14 & $\mathrm{~S}$ & 36.0550 & 13.9070 \\
\hline 15 & $\mathrm{~S}$ & 3.3470 & 16.5480 \\
\hline 16 & $\mathrm{C}$ & 0.3860 & 20.5120 \\
\hline 17 & $\mathrm{Q}$ & 0.0130 & 19.1140 \\
\hline 18 & $\mathrm{Q}$ & 0.0060 & 16.6110 \\
\hline 19 & $\mathrm{Y}$ & 0.0000 & 16.6110 \\
\hline 20 & $\mathrm{~L}$ & 0.0000 & 10.1030 \\
\hline 21 & $\mathrm{~K}$ & 0.0000 & 1.2080 \\
\hline 22 & $\mathrm{Q}$ & 0.0000 & 0.0000 \\
\hline
\end{tabular}

759 Fig. S5. (A) Predicted capsicumicine structure using the Phyre ${ }^{2}$ prediction server. (B) Aggregation structure predictions using TANGO (68) and SALSA algorithms (66). 